\title{
Combinatorics of Tesler matrices in the theory of parking functions and diagonal harmonics*
}

\author{
D. Armstrong, A. Garsia, J. Haglund, B. Rhohdes and B. Sagan
}

In [J. Haglund, A polynomial expression for the Hilbert series of the quotient ring of diagonal coinvariants, Adv. Math. 227 (2011) 2092-2106], the study of the Hilbert series of diagonal coinvariants is linked to combinatorial objects called Tesler matrices. In this paper we use operator identities from Macdonald polynomial theory to give new and short proofs of some of these results. We also develop the combinatorial theory of Tesler matrices and parking functions, extending results of P. Levande, and apply our results to prove various special cases of a positivity conjecture of Haglund.

KEYWORDS AND PHRASES: Parking functions, diagonal harmonics, tesler matrices.

\section{Introduction}

Tesler matrices have been with us since Alfred Young's work at the beginning of last century, only it took Glenn Tesler's work on the higher order Macdonald operators to bring them to his attention and into the Sloane online encyclopedia of integer sequences. To be precise, Tesler matrices emerge naturally from Young's "Rising operator" formula yielding the irreducible characters of the symmetric groups. In fact, the Frobenius image of Young's formula (see [14, p. 42]) may be written in the form

$$
s_{\mu}[X]=\left.\prod_{i=1}^{n} \Omega\left[z_{i} X\right] \prod_{1 \leq i<j \leq n}\left(1-z_{i} / z_{j}\right)\right|_{z_{n}^{\mu_{1}} z_{n-1}^{\mu_{2}} \cdots z_{1}^{\mu_{n}}}
$$

where, for any expression $E, \Omega[z E]$ denotes the generating function of the complete homogeneous symmetric functions plethystically evaluated at $E$.

${ }^{*}$ The first author is supported by NSF grant DMS-1001825. The second author is supported by NSF grant DMS-1068883. The third author is supported by NSF grant DMS-0901467. The fourth author is partially supported by NSF grant DMS1068861. 
(Readers unfamiliar with plethystic notation can consult [8, Chapter 1].) More explicitly

$$
\Omega[z E]=\sum_{m \geq 0} z^{m} h_{m}[E] .
$$

We should note also that Young's formula itself is but a specialization of the Raising operator formula for Hall-Littlewood functions $H_{\mu}(X ; t)$ (see $[14$, p. 213]) which may also be written in the form

$$
H_{\mu}[X ; t]=\left.\prod_{i=1}^{n} \Omega\left[z_{i} X\right] \prod_{1 \leq i<j \leq n} \frac{1-z_{i} / z_{j}}{1-t z_{i} / z_{j}}\right|_{z_{n}^{\mu_{1}} z_{n-1}^{\mu_{2}} \cdots z_{1}^{\mu_{n}}}
$$

Since we may write

$$
\frac{1-z_{i} / z_{j}}{1-t z_{i} / z_{j}}=\Omega\left[-\frac{z_{i}}{z_{j}}(1-t)\right]
$$

(1.3) becomes

$$
H_{\mu}[X ; t]=\left.\prod_{i=1}^{n} \Omega\left[z_{i} X\right] \prod_{1 \leq i<j \leq n} \Omega\left[-\frac{z_{i}}{z_{j}}(1-t)\right]\right|_{z_{n}^{\mu_{1}} z_{n-1}^{\mu_{2}} \cdots z_{1}^{\mu_{n}}}
$$

This in turn may be derived from N. Jing's "Vertex" operator formula for Hall-Littlewood functions. That is

$$
H_{\mu}[X ; t]=H_{\mu_{1}} H_{\mu_{2}} \cdots H_{\mu_{n}} 1
$$

where for any symmetric function $F[X]$ we set

$$
H_{a} F[X]=\left.F\left[X-\frac{1-t}{z}\right] \Omega[z X]\right|_{z^{a}} .
$$

In fact, note that this gives

$$
H_{b} H_{a} 1=\left.\Omega\left[z_{1}\left(X-\frac{1-t}{z_{2}}\right)\right] \Omega\left[z_{2} X\right]\right|_{z_{1}^{a} z_{2}^{b}}=\left.\Omega\left[z_{1} X\right] \Omega\left[z_{2} X\right] \Omega\left[-\frac{z_{1}}{z_{2}}(1-t)\right]\right|_{z_{1}^{a} z_{2}^{b}}
$$

and more generally we will have

$$
H_{a_{n}} \cdots H_{a_{2}} H_{a_{1}} 1=\left.\prod_{i=1}^{n} \Omega\left[z_{i} X\right] \prod_{1 \leq i<j \leq n} \Omega\left[-\frac{z_{i}}{z_{j}}(1-t)\right]\right|_{z_{1}^{a_{1}} z_{2}^{a_{2} \cdots z_{n}^{a_{n}}}}
$$

which shows that (1.4) and (1.5) are identical. 
Now note that the generic summand produced by the right-hand side of (1.7), after we expand all its factors according to (1.2) is

$$
\left.\prod_{i=1}^{n} z_{i}^{p_{i}} h_{p_{i}}[X] \prod_{1 \leq i<j \leq n}\left(z_{i} / z_{j}\right)^{p_{i j}} h_{p_{i j}}[-(1-t)]\right|_{z_{1}^{a_{1}} z_{2}^{a_{2} \ldots z_{n}^{a_{n}}}} .
$$

This forces the equalities

$$
p_{s}+\sum_{j=s+1}^{n} p_{s j}-\sum_{i=1}^{s-1} p_{i s}=a_{s} \quad(\text { for all } 1 \leq s \leq n) .
$$

We may thus associate to each summand an upper triangular matrix $A=$ $\left\|a_{i, j}\right\|_{i, j=1}^{n}$ by setting

$$
a_{i, j}= \begin{cases}p_{i} & \text { if } i=j \\ p_{i j} & \text { if } i<j \\ 0 & \text { otherwise }\end{cases}
$$

Denoting by $\mathcal{U P}$ the collection of upper triangular matrices with non-negative integer entries, let us set for a given integral vector $\left(a_{1}, a_{2}, \ldots, a_{n}\right)$

$$
\begin{aligned}
& \mathcal{T}\left(a_{1}, a_{2}, \ldots, a_{n}\right) \\
& \quad=\left\{A=\left\|a_{i, j}\right\|_{i, j=1}^{n} \in \mathcal{U P}: a_{s, s}+\sum_{j=s+1}^{n} a_{s, j}-\sum_{i=1}^{s-1} a_{i, s}=a_{s} \forall 1 \leq s \leq n\right\} .
\end{aligned}
$$

We will here and after refer to this as the collection of Tesler matrices with hook sums $\left(a_{1}, a_{2}, \ldots, a_{n}\right)$. For a given matrix $A=\left\|a_{i, j}\right\|_{i, j=1}^{n} \in \mathcal{U P}$ set

$$
w_{H L}(A)=\prod_{i=1}^{n} h_{a_{i, i}}[X] \prod_{1 \leq i<j \leq n} h_{a_{i, j}}[-(1-t)]
$$

and call it the "Hall-Littlewood weight" of $A$. With this notation (1.5) becomes

$$
H_{\mu}[X ; t]=\sum_{A \in \mathcal{T}\left(\mu_{n}, \mu_{n-1}, \ldots, \mu_{1}\right)} w_{H L}(A)
$$

In a recent paper Haglund [9], through Macdonald polynomial manipulations, was led to a new expression for the Hilbert series of the space of 
diagonal harmonics. By using Sloane's On-Line Encyclopedia of Integer Sequences, he was able to identify this new expression as a weighted sum of Tesler matrices. More precisely, Haglund obtained that

$$
\partial_{p_{1}}^{n} \nabla e_{n}=\left(-\frac{1}{M}\right)^{n} \sum_{A \in \mathcal{T}(1,1, \ldots, 1)} w_{H}(A)
$$

with

$$
w_{H}(A)=\prod_{i=1}^{n} h_{a_{i, i}}[-M] \prod_{1 \leq i<j \leq n} h_{a_{i, j}}[-M]
$$

where here and after it will be convenient to set

$$
M=(1-t)(1-q)
$$

In the same paper Haglund also proved that

$$
\partial_{p_{1}}^{n} \nabla^{m} e_{n}=\left(-\frac{1}{M}\right)^{n} \sum_{A \in \mathcal{T}(1, m, \ldots, m)} w_{H}(A) \quad(\text { for all } m \geq 1) .
$$

It is now important to note that the same manipulations that yielded (1.10) but applied in reverse yield that, in view of (1.12), the Haglund identity in (1.14) can also be written in the form

$$
\partial_{p_{1}}^{n} \nabla^{m} e_{n}=\left.\left(-\frac{1}{M}\right)^{n} \prod_{i=1}^{n} \Omega\left[-z_{i} M\right] \prod_{1 \leq i<j \leq n} \Omega\left[\frac{z_{i}}{z_{j}} M\right]\right|_{z_{1}^{1} z_{2}^{m} \cdots z_{n}^{m}}
$$

Note further that as long as the entries $\left(a_{1}, a_{2}, \ldots, a_{n}\right)$ are all positive each row of a matrix $A \in \mathcal{T}\left(a_{1}, a_{2}, \ldots, a_{n}\right)$ has to have at least one positive element, which is quite evident from the definition in (1.9). Thus, in view of $(1.12)$, in spite of the denominator factor $(-M)^{n}$, the expression

$$
P_{a_{1}, a_{2}, \ldots, a_{n}}(q, t)=\left(-\frac{1}{M}\right)^{n} \sum_{A \in \mathcal{T}\left(a_{1}, a_{2}, \ldots, a_{n}\right)} w_{H}(A)
$$

will necessarily evaluate to a polynomial. Further experimentations revealed that when $a_{1} \leq a_{2} \leq \cdots \leq a_{n}$ the polynomial $P_{a_{1}, a_{2}, \ldots, a_{n}}(q, t)$ turns out to have non-negative integer coefficients. All these findings spurred a flurry of research activities aimed at the identification of the structures $q, t$ counted by these polynomials and by what statistics. In particular the formulas in 
(1.11) and (1.14) beg to be reconciled with the previously conjectured formulas $[10,11]$ as weighted sums over parking functions and their natural m-extensions.

In this paper we present a variety of results which connect combinatorial properties of Tesler matrices to combinatorial properties of parking functions, diagonal harmonics and their closely related graded $S_{n}$ modules. Our hope is that this work will help form a base for future research on connections between these topics. We also give a new proof of the $m=1$ case of (1.15), as well as deriving some new results in the theory of plethystic Macdonald polynomial operators. Our exposition will start with the basic symmetric function identities of the subject and then present the combinatorial developments that followed. But before we can do that we need to collect a few identities of Macdonald polynomial theory which will turn out to be useful in our exposition.

\section{Our manipulatorial tool kit}

Let us recall that in [5] we have set

$$
\begin{array}{ll}
\text { a) } & D_{k} F[X]=\left.F\left[X+\frac{M}{z}\right] \Omega[-z X]\right|_{z^{k}} \quad \text { for }-\infty<k<+\infty . \\
\text { b) } \quad D_{k}^{*} F[X]=\left.F\left[X-\frac{\bar{M}}{z}\right] \Omega[z X]\right|_{z^{k}}
\end{array}
$$

Here " $\left.\right|_{z^{k}}$ " denotes the operation of taking the coefficient of $z^{k}$ in the preceding expression, $e_{m}$ and $h_{m}$ denote the elementary and homogeneous symmetric functions indexed by $m$, and for convenience we have set

$$
M=(1-t)(1-q), \quad \widetilde{M}=(1-1 / t)(1-1 / q) .
$$

Recall that the symmetric function operator $\nabla$ is defined by setting for the Macdonald basis $\left\{\tilde{H}_{\mu}(X ; q, t)\right\}_{\mu}$

$$
\nabla \tilde{H}_{\mu}=T_{\mu} \tilde{H}_{\mu} \quad\left(\text { with } T_{\mu}=t^{n(\mu)} q^{n\left(m u^{\prime}\right)} \text { and } n(\mu)=\sum_{i}(i-1) \mu_{i}\right) .
$$

The operators in $(2.1)$ are connected to $\nabla$ and the polynomials $\tilde{H}_{\mu}$ through the following basic identities:
(i) $\quad D_{0} \tilde{H}_{\mu}=-D_{\mu}(q, t) \tilde{H}_{\mu}$
$(i)^{*} \quad D_{0}^{*} \tilde{H}_{\mu}=-D_{\mu}(1 / q, 1 / t) \tilde{H}_{\mu}$
(ii) $D_{k} \underline{e}_{1}-\underline{e}_{1} D_{k}=M D_{k+1}$
$(\text { ii })^{*} \quad D_{k}^{*} \underline{e}_{1}-\underline{e}_{1} D_{k}^{*}=-\widetilde{M} D_{k+1}^{*}$
(iii)
$\nabla \underline{e}_{1} \nabla^{-1}=-D_{1}$
(iv) $\quad \nabla^{-1} \partial_{1} \nabla=\frac{1}{M} D_{-1}$
$(i i i)^{*}$
$\nabla D_{1}^{*} \nabla^{-1}=\underline{e}_{1}$
$(i v)^{*} \quad \nabla^{-1} D_{-1}^{*} \nabla=-\widetilde{M} \partial_{1}$ 
where $\underline{e}_{1}$ is simply the operator "multiplication by $e_{1}$ ", $\partial_{1}$ denotes its "Hall" scalar product adjoint and

$$
D_{\mu}(q, t)=M B_{\mu}(q, t)-1 \quad \text { with } B_{\mu}(q, t)=\sum_{c \in \mu} t^{l^{\prime}(c)} q^{a^{\prime}(c)} .
$$

Note that we also have

$$
\begin{aligned}
& \text { a) } D_{k} \partial_{1}-\partial_{1} D_{k}-D_{k-1} \text {, } \\
& \text { b) } D_{k}^{*} \partial_{1}-\partial_{1} D_{k}^{*}-D_{k-1}^{*} \text {. }
\end{aligned}
$$

Recall that for our version of the Macdonald polynomials the Macdonald Reciprocity formula states that

$$
\frac{\tilde{H}_{\alpha}\left[1+u D_{\beta}\right]}{\prod_{c \in \alpha}\left(1-u t^{l^{\prime}} q^{a^{\prime}}\right)}=\frac{\tilde{H}_{\beta}\left[1+u D_{\alpha}\right]}{\prod_{c \in \beta}\left(1-u t^{l^{\prime}} q^{a^{\prime}}\right)} \quad(\text { for all pairs } \alpha, \beta) .
$$

We will use here several special evaluations of (2.6). To begin, canceling the common factor $(1-u)$ out of the denominators on both sides of (2.6) then setting $u=1$ gives

$$
\frac{\tilde{H}_{\alpha}\left[M B_{\beta}\right]}{\Pi_{\alpha}}=\frac{\tilde{H}_{\beta}\left[M B_{\alpha}\right]}{\Pi_{\beta}} \quad(\text { for all pairs } \alpha, \beta) .
$$

On the other hand replacing $u$ by $1 / u$ and letting $u=0$ in (2.6) gives

$$
\left.(-1)^{|\alpha|} \frac{\tilde{H}_{\alpha}\left[D_{\beta}\right]}{T_{\alpha}}=(-1)^{|\beta|} \frac{\tilde{H}_{\beta}\left[D_{\alpha}\right]}{T_{\beta}} \quad \text { (for all pairs } \alpha, \beta\right) .
$$

Since for $\beta$ the empty partition we can take $\tilde{H}_{\beta}=1$ and $D_{\beta}=-1,(2.6)$ in this case reduces to

$$
\tilde{H}_{\alpha}[1-u]=\prod_{c \in \alpha}\left(1-u t^{l^{\prime}} q^{a^{\prime}}\right)=(1-u) \sum_{r=0}^{n-1}(-u)^{r} e_{r}\left[B_{\mu}-1\right] .
$$

This identity yields the coefficients of hook Schur functions in the expansion.

$$
\tilde{H}_{\mu}[X ; q, t]=\sum_{\lambda \vdash|\mu|} s_{\mu}[X] \tilde{K}_{\lambda \mu}(q, t) .
$$


Recall that the addition formula for Schur functions gives

$$
s_{\mu}[1-u]= \begin{cases}(-u)^{r}(1-u) & \text { if } \mu=\left(n-r, 1^{r}\right) \\ 0 & \text { otherwise. }\end{cases}
$$

Thus (2.10), with $X=1-u$, combined with (2.9) gives for $\mu \vdash n$

$$
\left\langle\tilde{H}_{\mu}, s_{\left(n-r, 1^{r}\right)}\right\rangle=e_{r}\left[B_{\mu}-1\right]
$$

and the identity $e_{r} h_{n-r}=s_{\left(n-r, 1^{r}\right)}+s_{\left(n-r-1,1^{r-1}\right)}$ gives

$$
\left\langle\tilde{H}_{\mu}, e_{r} h_{n-r}\right\rangle=e_{r}\left[B_{\mu}\right]
$$

Since for $\beta=(1)$ we have $\tilde{H}_{\beta}=1$ and $\Pi_{\beta}=1$, formula $(2.7)$ reduces to the surprisingly simple identity

$$
\tilde{H}_{\alpha}[M]=M B_{\alpha} \Pi_{\alpha}
$$

Last but not least we must also recall that we have the Pieri formulas

$$
\text { a) } e_{1} \tilde{H}_{\nu}=\sum_{\mu \leftarrow \nu} d_{\mu \nu} \tilde{H}_{\mu}, \quad \text { b) } e_{1}^{\perp} \tilde{H}_{\mu}=\sum_{\nu \rightarrow \mu} c_{\mu \nu} \tilde{H}_{\nu} .
$$

The final ingredients we need, to carry out our proofs, are the following summation formulas from [4] (the $k=1$ case of (2.17) occurs in unpublished work of M. Zabrocki)

$$
\sum_{\nu \rightarrow \mu} c_{\mu \nu}(q, t)\left(T_{\mu} / T_{\nu}\right)^{k}= \begin{cases}\frac{t q}{M} h_{k+1}\left[D_{\mu}(q, t) / t q\right] & \text { if } k \geq 1 \\ B_{\mu}(q, t) & \text { if } k=0\end{cases}
$$

$$
\sum_{\mu \leftarrow \nu} d_{\mu \nu}(q, t)\left(T_{\mu} / T_{\nu}\right)^{k}= \begin{cases}(-1)^{k-1} e_{k-1}\left[D_{\nu}(q, t)\right] & \text { if } k \geq 1 \\ 1 & \text { if } k=0\end{cases}
$$

Here $\nu \rightarrow \mu$ simply means that the sum is over $\nu$ 's obtained from $\mu$ by removing a corner cell and $\mu \leftarrow \nu$ means that the sum is over $\mu$ 's obtained from $\nu$ by adding a corner cell.

It will be useful to know that these two Pieri coefficients are related by the identity

$$
d_{\mu \nu}=M c_{\mu \nu} \frac{w_{\nu}}{w_{\mu}}
$$


Recall that our Macdonald Polynomials satisfy the orthogonality condition

$$
\left\langle\tilde{H}_{\lambda}, \tilde{H}_{\mu}\right\rangle_{*}=\chi(\lambda=\mu) w_{\mu}(q, t) .
$$

The *-scalar product, is simply related to the ordinary Hall scalar product by setting for all pairs of symmetric functions $f, g$

$$
\langle f, g\rangle_{*}=\langle f, \omega \phi g\rangle
$$

where it has been customary to let $\phi$ be the operator defined by setting for any symmetric function $f$

$$
\phi f[X]=f[M X]
$$

Note that the inverse of $\phi$ is usually written in the form

$$
f^{*}[X]=f[X / M]
$$

In particular we also have for all symmetric functions $f, g$

$$
\langle f, g\rangle=\left\langle f, \omega g^{*}\right\rangle_{*}
$$

The orthogonality relations in (1.15) yield the "Cauchy" identity for our Macdonald polynomials in the form

$$
\Omega\left[-\epsilon \frac{X Y}{M}\right]=\sum_{\mu} \frac{\tilde{H}_{\mu}[X] \tilde{H}_{\mu}[Y]}{w_{\mu}}
$$

which restricted to its homogeneous component of degree $n$ in $X$ and $Y$ reduces to

$$
e_{n}\left[\frac{X Y}{M}\right]=\sum_{\mu \vdash n} \frac{\tilde{H}_{\mu}[X] \tilde{H}_{\mu}[Y]}{w_{\mu}} .
$$

Note that the orthogonality relations in (2.19) yield us the following Macdonald polynomial expansions

Proposition 2.1. For all $n \geq 1$ we have
a) $e_{n}\left[\frac{X}{M}\right]=\sum_{\mu \vdash n} \frac{\tilde{H}_{\mu}[X]}{w_{\mu}}$
b) $h_{k}\left[\frac{X}{M}\right] e_{n-k}\left[\frac{X}{M}\right]=\sum_{\mu \vdash n} \frac{e_{k}\left[B_{\mu}\right] \tilde{H}_{\mu}[X]}{w_{\mu}}$ 


$$
\begin{gathered}
\text { c) } h_{n}\left[\frac{X}{M}\right]=\sum_{\mu \vdash n} \frac{T_{\mu} \tilde{H}_{\mu}[X]}{w_{\mu}} \\
\text { d) }(-1)^{n-1} p_{n}=\left(1-t^{n}\right)\left(1-q^{n}\right) \sum_{\mu \vdash n} \frac{\Pi_{\mu} \tilde{H}_{\mu}[X]}{w_{\mu}} \\
\text { e) } e_{1}[X / M]^{n}=\sum_{\mu \vdash n} \frac{\tilde{H}_{\mu}[X]}{w_{\mu}}\left\langle\tilde{H}_{\mu}, e_{1}^{n}\right\rangle \\
f) \quad e_{n}=\sum_{\mu \vdash m} \frac{\tilde{H}_{\mu}[X] M B_{\mu} \Pi_{\mu}}{w_{\mu}} .
\end{gathered}
$$

Finally it is good to keep in mind, for future use, that we have for all partitions $\mu$

$$
T_{\mu} \omega \tilde{H}_{\mu}[X ; 1 / q 1 /, t]=\tilde{H}_{\mu}[X ; q, t]
$$

Remark 2.1. It was conjectured in [4] and proved in [11] that the bigraded Frobenius characteristic of the diagonal Harmonics of $S_{n}$ is given by the symmetric function

$$
D H_{n}[X ; q, t]=\sum_{\mu \vdash n} \frac{T_{\mu} \tilde{H}_{\mu}(X ; q, t) M B_{\mu}(q, t) \Pi_{\mu}(q, t)}{w_{\mu}(q, t)} .
$$

Surprisingly the intricate rational function on the right-hand side is none other than $\nabla e_{n}$. To see this we simply combine the relation in (2.14) with the degree $n$ restricted Macdonald-Cauchy formula (2.25) obtaining

$$
e_{n}[X]=e_{n}\left[\frac{X M}{M}\right]=\sum_{\mu \vdash n} \frac{\tilde{H}_{\mu}[X] M B_{\mu} \Pi_{\mu}}{w_{\mu}} .
$$

This is perhaps the simplest way to prove (2.26)f). This discovery is precisely what led to the introduction of $\nabla$ in the first place.

\section{The Hilbert series of diagonal harmonics}

Our first goal here is to obtain new proofs of the results in [9] by using the connection between Tesler matrices and plethystic operators. The basic ingredient in this approach is provided by the following 
Proposition 3.1. For any symmetric function $F[X]$ and any sequence of integers $a_{1}, a_{2}, \ldots, a_{n}$ we have

$$
\begin{aligned}
D_{a_{n}} & \left.\cdots D_{a_{2}} D_{a_{1}} F[X]\right|_{X=M} \\
= & \left.F\left[M+\sum_{i=1}^{n} \frac{M}{z_{i}}\right] \prod_{i=1}^{n} \Omega\left[-z_{i} M\right] \prod_{1 \leq i<j \leq n} \Omega\left[\frac{z_{i}}{z_{j}} M\right]\right|_{z_{1}^{a_{1}} z_{2}^{a_{2} \ldots z_{n}}} \\
= & F\left[M+\sum_{i=1}^{n} \frac{M}{z_{i}}\right] \prod_{i=1}^{n} \frac{\left(1-z_{i}\right)\left(1-t q z_{i}\right)}{\left(1-t z_{i}\right)\left(1-q z_{i}\right)} \\
& \times\left.\prod_{1 \leq i<j \leq n} \frac{\left(1-\frac{z_{i}}{z_{j}}\right)\left(1-t q \frac{z_{i}}{z_{j}}\right)}{\left(1-t \frac{z_{i}}{z_{j}}\right)\left(1-q \frac{z_{i}}{z_{j}}\right)}\right|_{z_{1}^{a_{1}} z_{2}^{a_{2} \ldots z_{n}^{a_{n}}}} .
\end{aligned}
$$

Proof. The definition in (2.1)a) gives

$$
D_{a_{1}} F[X]=\left.F\left[X+\frac{M}{z_{1}}\right] \Omega\left[-z_{1} X\right]\right|_{z_{1}^{a_{1}}}
$$

and using (2.1)a) again we get

$$
\begin{aligned}
D_{a_{2}} D_{a_{1}} F[X] & =\left.F\left[X+\frac{M}{z_{1}}+\frac{M}{z_{2}}\right] \Omega\left[-z_{1}\left(X+\frac{M}{z_{2}}\right)\right] \Omega\left[-z_{2} X\right]\right|_{z_{1}^{a_{1}} z_{2}^{a_{2}}} \\
& =\left.F\left[X+\frac{M}{z_{1}}+\frac{M}{z_{2}}\right] \Omega\left[-\frac{z_{1}}{z_{2}} M\right] \Omega\left[-z_{1} X-z_{2} X\right]\right|_{z_{1}^{a_{1}} z_{2}^{a_{2}}} .
\end{aligned}
$$

As before, this should make it clear that the successive actions of $D_{a_{3}} \cdots D_{a_{k}}$ will eventually yield the identity

$$
\begin{aligned}
D_{a_{n}} & \cdots D_{a_{2}} D_{a_{1}} F[X] \\
= & F\left[X+\sum_{i=1}^{n} \frac{M}{z_{i}}\right] \prod_{1 \leq i<j \leq n} \Omega\left[-\frac{z_{i}}{z_{j}} M\right] \\
& \times\left.\Omega\left[-z_{1} X-z_{2} X-\cdots-z_{n} X\right]\right|_{z_{1}^{a_{1}} z_{2}^{a_{2} \cdots z_{n}^{a_{n}}}} .
\end{aligned}
$$

Setting $X=M$ gives the first equality in (1.6), but then the second equality holds as well since for any monomial $v$ we have

$$
\Omega[-v M]=\Omega[v t+v q-v-q t v]=\frac{(1-v)(1-q t v)}{(1-t v)(1-q v)} .
$$

The identity in (3.1) has the following important corollary. 


\section{Theorem 3.1.}

$$
\partial_{p_{1}}^{n} \nabla^{m} e_{n}=\left.\left(-\frac{1}{M}\right)^{n} D_{m-1}^{n-1} D_{0} e_{n+1}\left[\frac{X}{M}\right]\right|_{X=M}
$$

Proof. Setting $a_{0}=0, a_{2}=a_{3}=\cdots=a_{n}=m-1$ and $F[X]=e_{n+1}\left[\frac{X}{M}\right]$ in (3.1) gives

$$
\left.D_{m-1}^{n-1} D_{0} e_{n+1}\left[\frac{X}{M}\right]\right|_{X=M}=\left.\prod_{i=1}^{n} \Omega\left[-z_{i} M\right] \prod_{1 \leq i<j \leq n} \Omega\left[\frac{z_{i}}{z_{j}} M\right]\right|_{z_{1}^{1} z_{2}^{m} \cdots z_{n}^{m}}
$$

because in this case we have

$$
F\left[M+\sum_{i=1}^{n} \frac{M}{z_{i}}\right]=e_{n+1}\left[1+\frac{1}{z_{1}}+\frac{1}{z_{2}}+\cdots+\frac{1}{z_{n}}\right]=\frac{1}{z_{1} z_{2} \cdots z_{n}} .
$$

Combining (3.3) with (1.15) shows that (3.2) is simply another way of writing Haglund's identity (1.11).

Our challenge here is to give a direct proof of (3.2). We have so far succeeded in carrying this out for $m=1$. Research to obtain the general case is still in progress. The rest of this section is dedicated to the proof of the case $m=1$ of (3.2) and the derivation of another Tesler matrix formula for the Hilbert series of diagonal harmonics. We will start with the latter formula since it requires the least amount of machinery.

Theorem 3.2. For all $n \geq 1$ we have

$$
\begin{aligned}
\partial_{p_{1}}^{n-1} \nabla e_{n-1} & =\left(-\frac{1}{M}\right)^{n-1} D_{-1}^{n} p_{n}\left[\frac{X}{M}\right] \\
& =\left.\left(-\frac{1}{M}\right)^{n-1} \prod_{i=1}^{n} \Omega\left[-z_{i} M\right] \prod_{1 \leq i<j \leq n} \Omega\left[-\frac{z_{i}}{z_{j}} M\right]\right|_{z_{1}^{n-1} z_{2}^{-1} \cdots z_{n}^{-1}}
\end{aligned}
$$

In particular it follows that

$$
\partial_{p_{1}}^{n-1} \nabla e_{n-1}=\left(-\frac{1}{M}\right)^{n-1} \sum_{A \in \mathcal{T}(n-1,-1,-1, \ldots,-1)} w_{H}[A] .
$$

Proof. The definition of $D_{-1}$ gives that

$$
D_{-1} p_{n}\left[\frac{X}{M}\right]=\left.\left(p_{n}\left[\frac{X}{M}\right]+\frac{1}{z^{n}}\right) \Omega[-z X]\right|_{z^{-1}}=(-1)^{n-1} e_{n-1}
$$


Using this and (2.3)(iv) we derive that

$$
\begin{gathered}
\left(-\frac{1}{M}\right)^{n-1} D_{-1}^{n} p_{n}\left[\frac{X}{M}\right]=\left(\frac{1}{M}\right)^{n-1} D_{-1}^{n-1} e_{n-1} \\
=\nabla^{-1} \partial_{p_{1}}^{n-1} \nabla e_{n-1}=\partial_{p_{1}}^{n-1} \nabla e_{n-1} .
\end{gathered}
$$

This proves the first equality in (3.4).

But Proposition 3.1 with $a_{1}=a_{2}=\cdots=a_{n}=-1$ gives

$$
\left.D_{-1}^{n} p_{n}\left[\frac{X}{M}\right]\right|_{X=M}=\left.\left(1+\sum_{i=1}^{n} \frac{1}{z_{i}^{n}}\right) \prod_{i=1}^{n} \Omega\left[-z_{i} M\right] \prod_{1 \leq i<j \leq n} \Omega\left[-\frac{z_{i}}{z_{j}} M\right]\right|_{z_{1}^{-1} z_{2}^{-1} \cdots z_{n}^{-1}}
$$

and (3.6) gives

$$
\begin{aligned}
\partial_{p_{1}}^{n-1} \nabla e_{n-1}= & \left(-\frac{1}{M}\right)^{n-1}\left(1+\sum_{i=1}^{n} \frac{1}{z_{i}^{n}}\right) \\
& \times\left.\prod_{i=1}^{n} \Omega\left[-z_{i} M\right] \prod_{1 \leq i<j \leq n} \Omega\left[-\frac{z_{i}}{z_{j}} M\right]\right|_{z_{1}^{-1} z_{2}^{-1} \cdots z_{n}^{-1}}
\end{aligned}
$$

Note next that if we set $F=1$ and $a_{1}=a_{2}=\cdots=a_{n}=-1$ in (3.1) we obtain

$$
0=\left.\left.D_{-1}^{n} 1\right|_{X=\bar{M}} \prod_{i=1}^{n} \Omega\left[-z_{i} M\right] \prod_{1 \leq i<j \leq n} \Omega\left[-\frac{z_{i}}{z_{j}} M\right]\right|_{z_{1}^{-1} z_{2}^{-1} \cdots z_{n}^{-1}}
$$

that eliminates one of the terms in (3.8). We claim that only the term yielded by $\frac{1}{z_{1}^{n}}$ survives. That is we have

$$
\left.\frac{1}{z_{k}^{n}} \prod_{i=1}^{n} \Omega\left[-M z_{i}\right] \prod_{1 \leq i<j \leq n} \Omega\left[-M z_{i} / z_{j}\right]\right|_{z_{1}^{-1} z_{2}^{-1} \cdots z_{n}^{-1}}=0 \quad(\text { for all } 2 \leq k \leq n) .
$$

Let us now rewrite the LHS in the expanded form, that is

$$
\left.\frac{1}{z_{k}^{n}} \prod_{i=1}^{n} \sum_{p_{i} \geq 0} z_{i}^{p_{i}} h_{p_{i}}[-M] \prod_{1 \leq i<j \leq n} \sum_{r_{i, j} \geq 0} \frac{z_{i}^{r_{i, j}}}{z_{j}^{r_{i, j}}} h_{r_{i, j}}[-M]\right|_{z_{1}^{-1} z_{2}^{-1} \cdots z_{n}^{-1}} .
$$

The exponent of $z_{1}$ in the generic term of the product of these geometric series must satisfy the equation

$$
p_{1}+\sum_{j=2}^{n} r_{1, j}=-1
$$


This is, of course impossible, causing (3.10) to be true precisely as asserted. Using (3.9) and (3.10) in (3.8) proves the second equality in (3.4), completing our proof.

Our proof of (3.2) for $m=1$ is more elaborate and requires the following auxiliary identity.

Proposition 3.2. For any symmetric function $F[X]$ we have

$$
\sum_{\mu \vdash n+1} \frac{M B_{\mu} \Pi_{\mu}}{w_{\mu}} F\left[M B_{\mu}\right]=\left.\Delta_{e_{n}} F[X]\right|_{X=M}
$$

Proof. We need only prove this for $F[X]=\tilde{H}_{\gamma}[X]$ for arbitrary $\gamma$. In this case (3.11) becomes

$$
\sum_{\mu \vdash n+1} \frac{M B_{\mu} \Pi_{\mu}}{w_{\mu}} \tilde{H}_{\gamma}\left[M B_{\mu}\right]=\tilde{H}_{\gamma}[M] e_{n}\left[B_{\gamma}\right]=M B_{\gamma} \Pi_{\gamma} e_{n}\left[B_{\gamma}\right] .
$$

Since by the reciprocity in (2.7) we have

$$
\frac{\tilde{H}_{\gamma}\left[M B_{\mu}\right]}{\Pi_{\gamma}}=\frac{\tilde{H}_{\mu}\left[M B_{\gamma}\right]}{\Pi_{\mu}}
$$

(3.12) becomes

$$
\Pi_{\gamma} \sum_{\mu \vdash n+1} \frac{M B_{\mu}}{w_{\mu}} \tilde{H}_{\mu}\left[M B_{\gamma}\right]=M B_{\gamma} \Pi_{\gamma} e_{n}\left[B_{\gamma}\right]
$$

or more simply

$$
\sum_{\mu \vdash n+1} \frac{M B_{\mu}}{w_{\mu}} \tilde{H}_{\mu}\left[M B_{\gamma}\right]=M B_{\gamma} e_{n}\left[B_{\gamma}\right]
$$

But recall that we have

$$
B_{\mu}=\sum_{\nu \rightarrow \mu} c_{\mu \nu}
$$

and (1.3) becomes

$$
\sum_{\mu \vdash n+1} \frac{M}{w_{\mu}} \tilde{H}_{\mu}\left[M B_{\gamma}\right] \sum_{\nu \rightarrow \mu} c_{\mu \nu}=M B_{\gamma} e_{n}\left[B_{\gamma}\right]
$$


Now for the left-hand side we have

$$
\begin{aligned}
L H S & =\sum_{\nu \vdash n} \frac{1}{w_{\nu}} \sum_{\mu \leftarrow \nu} \frac{M w_{\nu}}{w_{\mu}} c_{\mu \nu} \tilde{H}_{\mu}\left[M B_{\gamma}\right] \\
& =\sum_{\nu \vdash n} \frac{1}{w_{\nu}} \sum_{\mu \leftarrow \nu} d_{\mu \nu} \tilde{H}_{\mu}\left[M B_{\gamma}\right] \\
& =\sum_{\nu \vdash n} \frac{1}{w_{\nu}} e_{1}\left[M B_{\gamma}\right] \tilde{H}_{\nu}\left[M B_{\gamma}\right] \\
& =e_{1}\left[M B_{\gamma}\right] e_{n}\left[\frac{M B_{\gamma}}{M}\right]=M B_{\gamma} e_{n}\left[B_{\gamma}\right]=\operatorname{RHS}(! ! ! !)
\end{aligned}
$$

and our proof is complete.

As a corollary we obtain

Proposition 3.3. For $F \in \Lambda^{=k}$ with $k \leq n$ we have

$$
\sum_{\mu \vdash n+1} \frac{M B_{\mu} \Pi_{\mu}}{w_{\mu}} F\left[M B_{\mu}\right]= \begin{cases}\left.\nabla F[X]\right|_{X=M} & \text { if } k=n \\ 0 & \text { if } k<n .\end{cases}
$$

Proof. From (3.11) we get that the left hand side of (3.15) is

$$
\left.\Delta_{e_{n}} F[X]\right|_{X=M}
$$

but for a symmetric function $F[X]$ of degree $n$ we have $\Delta_{e_{n}} F[X]=\nabla F[X]$, thus the first alternative in (1.5) is immediate. On the other hand for $k<n$ the expansion of $F$ in the Macdonald basis will involve $\tilde{H}_{\gamma}$ 's with $\gamma \vdash k$ and even before we make the evaluation at $X=M$ the identity

$$
\Delta_{e_{n}} \tilde{H}_{\gamma}[X]=e_{n}\left[B_{\gamma}\right] \tilde{H}_{\gamma}[S]=0 \quad(\text { for all } \gamma \vdash k<n)
$$

forces $\Delta_{e_{n}} F=0$, yielding the second alternative in (3.15).

We are now in a position to give a new and direct proof of (3.2) for $m=1$.

\section{Theorem 3.3.}

$$
\partial_{p_{1}}^{n} \nabla e_{n}=\left.\left(-\frac{1}{M}\right)^{n} D_{0}^{n} e_{n+1}\left[\frac{X}{M}\right]\right|_{X=M}
$$


Proof. From (2.26)a) and (2.3)(i) we get

$$
\begin{aligned}
\left.D_{0}^{n} e_{n+1}\left[\frac{X}{M}\right]\right|_{X=M} & =\left.\left(D_{0}^{n} \sum_{\mu \vdash n+1} \frac{\tilde{H}_{\mu}[X ; q, t]}{w_{\mu}}\right)\right|_{X=M} \\
& =\left.\sum_{\mu \vdash n+1} \frac{\tilde{H}_{\mu}[X ; q, t]\left(1-M B_{\mu}\right)^{n}}{w_{\mu}}\right|_{X=M} \\
& =\sum_{k=0}^{n}\left(\begin{array}{l}
n \\
k
\end{array}\right)(-M)^{k} \sum_{\mu \vdash n+1} \frac{M B_{\mu} \Pi_{\mu}}{w_{\mu}} B_{\mu}^{k},
\end{aligned}
$$

and Proposition 3.3 with $F=e_{1}\left[\frac{X}{M}\right]^{k}$ and $0 \leq k \leq n$ gives

$$
\left.\left(-\frac{1}{M}\right)^{n} D_{0}^{n} e_{n+1}\left[\frac{X}{M}\right]\right|_{X=M}=\left.\nabla e_{1}^{n}\left[\frac{X}{M}\right]\right|_{X=M}
$$

(by $(2.26)$ e) and the definition of $\left.\nabla)=\sum_{\mu \vdash n} \frac{T_{\mu} \tilde{H}_{\mu}[X]}{w_{\mu}}\left\langle\tilde{H}_{\mu}, e_{1}^{n}\right\rangle\right]\left.\right|_{X=M}$

$$
(\operatorname{by}(2.14))=\sum_{\mu \vdash n} \frac{T_{\mu} M B_{\mu} \Pi_{\mu}}{w_{\mu}}\left\langle\tilde{H}_{\mu}, e_{1}^{n}\right\rangle
$$

(by (2.26)f) and $\left.\partial_{p_{1}}=e_{1}^{\perp}\right)=\partial_{p_{1}}^{n} \nabla e_{n}$.

This proves (3.16) and completes our argument.

Remark 3.1. Note that the identity in (3.2) for $m=2$ becomes

$$
\partial_{p_{1}}^{n} \nabla^{2} e_{n}=\left.\left(-\frac{1}{M}\right)^{n} D_{1}^{n-1} D_{0} e_{n+1}\left[\frac{X}{M}\right]\right|_{X=M}
$$

Since $(2.3)$ (iii) gives $D_{1}=-\nabla e_{1} \nabla^{-1}$ we can rewrite this as

$$
\partial_{p_{1}}^{n} \nabla^{2} e_{n}=-\left.\left(\frac{1}{M}\right)^{n} \nabla e_{1}^{n-1} \nabla^{-1} D_{0} e_{n+1}\left[\frac{X}{M}\right]\right|_{X=M}
$$

which is a really surprising formula, since it shows that there are identities for these operators that still remain to be discovered.

Remark 3.2. Computer experimentation has shown that $(-1)^{n-1} \partial_{p_{1}}^{n} \nabla^{-m} e_{n}$ is a polynomial in $\mathrm{Q}[1 / q, 1 / t]$ with non negative integer coefficients. This phenomenon can be explained by means of the operator " $\downarrow$ " which is defined by setting for any symmetric polynomial $F[X ; q, t]$

$$
\downarrow F[X ; q, t]=\omega F[X ; 1 / q, 1 / t] .
$$


In fact, note that for $F=\tilde{H}_{\mu}[X ; q, t](2.27)$ gives

$$
\downarrow \tilde{H}_{\mu}[X ; q, t]=\omega \tilde{H}_{\mu}[X ; 1 / q, 1 / t]=\frac{1}{T_{\mu}} \tilde{H}_{\mu}[X ; q, t] .
$$

Thus (2.27) again gives

$$
\downarrow \nabla \downarrow \tilde{H}_{\mu}[X ; q, t]=\downarrow \tilde{H}_{\mu}[X ; q, t]=\frac{1}{T_{\mu}} \tilde{H}_{\mu}[X ; q, t]=\nabla^{-1} \tilde{H}_{\mu}[X ; q, t]
$$

and since the operator $\downarrow \nabla \downarrow$ is linear in the linear span of the basis $\left\{\tilde{H}_{\mu}\right\}_{\mu}$ it follows that

$$
\downarrow \nabla \downarrow=\nabla^{-1} \text {. }
$$

Now this implies that

$$
(-1)^{n-1} \nabla^{-m} e_{n}[X]=\downarrow \nabla^{m}(-1)^{n-1} h_{n}[X]
$$

and the Schur positivity of $(-1)^{n-1} \nabla^{-m} e_{n}[X]$ now follows from the conjectured Schur positivity of $\nabla^{m}(-1)^{n-1} h_{n}[X]$. Since the operators $\partial_{p_{1}}$ and $\downarrow$ commute we see then that applying $\downarrow$ to both sides of (1.14) we derive that $(3.21)$

$$
\partial_{p_{1}}^{n} \nabla^{-m} h_{n}=\left.\left(-\frac{t q}{M}\right)^{n} \sum_{A \in \mathcal{T}(1, m, \ldots, m)} w_{H}(A)\right|_{t=1 / t, q=1 / q} \quad(\text { for all } m \geq 1) .
$$

Remark 3.3. Note that using the operator $\downarrow$ we can derive from (2.26)f) that

$$
h_{n}=\sum_{\mu \vdash m} \frac{\omega \tilde{H}_{\mu}[X ; 1 / q, 1 / t](1-1 / t)(1-1 / q) B_{\mu}(1 / q, 1 / t) \Pi_{\mu}(1 / q, 1 / t)}{w_{\mu}(1 / q, 1 / t)} .
$$

Since by definition we have

$$
\begin{aligned}
& w_{\mu}(q, t)=\prod_{c \in \mu}\left(q^{a(c)}-t^{l(c)+1}\right)\left(t^{l(c)}-q^{a(c)+1}\right) \\
& \Pi_{\mu}(q, t)=\prod_{c \in \mu c \neq(0,0)}\left(1-q^{a^{\prime}(c)} t^{l^{\prime}(c)}\right) .
\end{aligned}
$$


Thus

$$
\begin{gathered}
w_{\mu}(1 / q, 1 / t)=\prod_{c \in \mu}\left(q^{-a(c)}-t^{-l(c)-1}\right)\left(t^{-l(c)}-q^{-a(c)-1}\right), \\
\Pi_{\mu}(1 / q, 1 / t)=\prod_{c \in \mu c \neq(0,0)}\left(1-q^{-a^{\prime}(c)} t^{-l^{\prime}(c)}\right) \\
w_{\mu}(1 / q, 1 / t)=(q t)^{-n} T_{\mu}^{-2} w_{\mu}(q, t), \quad \Pi_{\mu}(1 / q, 1 / t)=(-1)^{n-1} T_{\mu}^{-1} \Pi_{\mu}(q, t)
\end{gathered}
$$

and (3.22) becomes

$$
h_{n}=(-q t)^{n-1} \sum_{\mu \vdash m} \frac{\tilde{H}_{\mu}[X ; q, t] M B_{\mu}(1 / q, 1 / t) \Pi_{\mu}(q, t)}{w_{\mu}(q, t)} .
$$

It should be noted that other surprising identities may be obtained by means of the operator $\downarrow$. For instance applying it to both sides of $\partial_{p_{1}} \tilde{H}_{\mu}(X ; q, t)=\sum_{\nu \rightarrow \mu} c_{\mu \nu}(q, t) \tilde{H}_{\nu}[X ; q, t)$ gives by $(3.19)$

$$
\partial_{p_{1}} \frac{1}{T_{\mu}} \tilde{H}_{\mu}[X ; q, t]=\sum_{\nu \rightarrow \mu} c_{\mu \nu}(1 / q, 1 / t) \frac{1}{T_{\nu}} \tilde{H}_{\nu}[X ; q, t]
$$

from which we derive that $c_{\mu \nu}(1 / q, 1 / t)=\frac{T_{\nu}}{T_{\mu}} c_{\mu \nu}(q, t)$. An application of $\downarrow$ to the identity $B_{\mu}(q, t)=\sum_{\nu \rightarrow \mu} c_{\mu \nu}(q, t)$ gives

$$
T_{\mu} B_{\mu}(1 / q, 1 / t)=\sum_{\nu \rightarrow \mu} c_{\mu \nu}(q, t) T_{\nu}
$$

which for $\mu \vdash n$ can also be rewritten as

$$
e_{n-1}\left[B_{\mu}(q, t)\right]=\sum_{\nu \rightarrow \mu} c_{\mu \nu}(q, t) T_{\nu}
$$

\section{Further remarkable $D_{0}$ identities}

In this section we will explore some of the consequences obtained by combining the action of $D_{o}$ with the following identity of Haglund [7].

Proposition 4.1. For any positive integers $m, n$ and any $P \in \Lambda^{n}$, we have

$$
\left\langle\Delta_{e_{m-1}} e_{n}, P\right\rangle=\left\langle\Delta_{\omega P} e_{m}, s_{m}\right\rangle
$$


Proof. The operator $\Delta_{e_{m-1}}$ applied to both sides of (2.26) gives

$$
\Delta_{e_{m-1}} e_{n}=\sum_{\mu \vdash n} \frac{M B_{\mu} \Pi_{\mu} \tilde{H}_{\mu}(x ; q, t)}{w_{\mu}} e_{m-1}\left[B_{\mu}\right]
$$

Using again (2.26) with $n \rightarrow m$ we also have

$$
\Delta_{\omega P} e_{m}=\sum_{\alpha \vdash m} \frac{M B_{\alpha} \Pi_{\alpha} \tilde{H}_{\alpha}(x ; q, t)}{w_{\alpha}}(\omega P)\left[B_{\alpha}\right] .
$$

Using (4.2) and (4.3) we get the explicit form of (4.1), which is

$$
\sum_{\mu \vdash n} \frac{M B_{\mu} \Pi_{\mu} e_{m-1}\left[B_{\mu}\right]}{w_{\mu}}\left\langle\tilde{H}_{\mu}, P\right\rangle=\sum_{\alpha \vdash m} \frac{M B_{\alpha} \Pi_{\alpha}}{w_{\alpha}}(\omega P)\left[B_{\alpha}\right] .
$$

This given, our idea here as in [7], is to establish (4.1) by checking its validity when $P$ varies among all the members of a symmetric function basis. It turns out that a simpler proof of (4.1) is obtained by testing (4.3) with the modified Macdonald basis

$$
\left\{\frac{\omega \tilde{H}_{\gamma}[M X ; q, t]}{w_{\gamma}}\right\}_{\gamma}
$$

The source of the simplification is due to the fact that this basis is precisely the Hall scalar product dual of the Macdonald basis $\left\{\tilde{H}_{\gamma}[X]\right\}_{\gamma}$. Using this fact, putting $P=\frac{\omega \tilde{H}_{\gamma}[M X]}{w_{\gamma}}$ in (4.4) gives

$$
\frac{M B_{\gamma} \Pi_{\gamma} e_{m-1}\left[B_{\gamma}\right]}{w_{\gamma}}=\sum_{\alpha \vdash m} \frac{M B_{\alpha} \Pi_{\alpha}}{w_{\alpha}} \frac{\tilde{H}_{\gamma}\left[M B_{\alpha}\right]}{w_{\gamma}} .
$$

Carrying out the simplifications this may be rewritten as

$$
B_{\gamma} e_{m-1}\left[B_{\gamma}\right]=\sum_{\alpha \vdash m} \frac{B_{\alpha} \Pi_{\alpha}}{w_{\alpha}} \frac{\tilde{H}_{\gamma}\left[M B_{\alpha}\right]}{\Pi_{\gamma}}
$$

and Macdonald reciprocity reduces this to

$$
B_{\gamma} e_{m-1}\left[B_{\gamma}\right]=\sum_{\alpha \vdash m} \frac{B_{\alpha}}{w_{\alpha}} \tilde{H}_{\alpha}\left[M B_{\gamma}\right] .
$$


Now we may rewrite $(2.16)$ with $k=0$, as

$$
B_{\alpha}=\sum_{\beta \rightarrow \alpha} c_{\alpha \beta}
$$

Using this in the right hand side of (4.5) gives

$$
R H S=\sum_{\alpha \vdash m} \frac{\tilde{H}_{\alpha}\left[M B_{\gamma}\right]}{w_{\alpha}} \sum_{\beta \rightarrow \alpha} c_{\alpha \beta}=\sum_{\beta \vdash m-1} \frac{1}{w_{\beta}} \sum_{\alpha \leftarrow \beta} \tilde{H}_{\alpha}\left[M B_{\gamma}\right] c_{\alpha \beta} \frac{w_{\beta}}{w_{\alpha}} .
$$

Next we use (2.18), in the form

$$
c_{\alpha \beta} \frac{w_{\beta}}{w_{\alpha}}==\frac{1}{M} d_{\alpha \beta}
$$

and (4.7) becomes

$$
\begin{aligned}
R H S & =\frac{1}{M} \sum_{\beta \vdash m-1} \frac{1}{w_{\beta}} \sum_{\alpha \leftarrow \beta} \tilde{H}_{\alpha}\left[M B_{\gamma}\right] d_{\alpha \beta} \\
& =\frac{1}{M} \sum_{\beta \vdash m-1} \frac{M B_{\gamma} \tilde{H}_{\beta}\left[M B_{\gamma}\right]}{w_{\beta}} \\
& =B_{\gamma} \sum_{\beta \vdash m-1} \frac{\tilde{H}_{\beta}\left[M B_{\gamma} ; q, t\right]}{w_{\beta}} \\
(\text { by (2.26)a)) } & =B_{\gamma} e_{m-1}\left[\frac{M B_{\gamma}}{M}\right]=B_{\gamma} e_{m-1}\left[B_{\gamma}\right]
\end{aligned}
$$

proving (4.5) as desired.

Study of the above proof led us to the following variation of (4.1).

\section{Proposition 4.2.}

$$
(-q t)^{m-1}\left\langle\Delta_{h_{m-1}} e_{n}, P\right\rangle=\left\langle\Delta_{\omega P} s_{m}, s_{1^{m}}\right\rangle .
$$

Proof. As we did for (4.1), we will prove (4.8) by testing it with $P=$ $\omega \tilde{H}_{\gamma}[M X] / w_{\gamma}$. To this end note first that the left-hand side is

$$
L H S=(-q t)^{m-1} \sum_{\mu \vdash n} \frac{M B_{\mu} \Pi_{\mu} h_{m-1}\left[B_{\mu}\right]}{w_{\mu}}\left\langle\tilde{H}_{\mu}, P\right\rangle
$$

and the right-hand side (using (3.23)) is

$$
R H S=(-q t)^{m-1} \sum_{\mu \vdash m} \frac{M \tilde{B}_{\mu} \Pi_{\mu}}{w_{\mu}}(\omega P)\left[B_{\mu}\right] .
$$


With this choice of $P$ we get

$$
L H S=(-q t)^{m-1} \frac{M B_{\gamma} \Pi_{\gamma} h_{m-1}\left[B_{\gamma}\right]}{w_{\gamma}}
$$

and

$$
R H S=(-q t)^{m-1} \sum_{\mu \vdash m} \frac{M \tilde{B}_{\mu} \Pi_{\mu} T_{\mu}}{w_{\mu}} \frac{\tilde{H}_{\gamma}\left[M B_{\mu}\right]}{w_{\gamma}} .
$$

We are thus reduced to showing, upon omission of the common factor $(-q t)^{m-1}$, division by $M \Pi_{\gamma} / w_{\gamma}$ and setting $\tilde{B}_{\mu}=B_{\mu}(1 / q, 1 / t)$,

$$
B_{\gamma} h_{m-1}\left[B_{\gamma}\right]=\sum_{\mu \vdash m} \frac{\tilde{B}_{\mu} \Pi_{\mu} T_{\mu}}{w_{\mu}} \frac{\tilde{H}_{\gamma}\left[M B_{\mu}\right]}{\Pi_{\gamma}} .
$$

The next idea is to use the identity in (3.24), namely

$$
T_{\mu} \tilde{B}_{\mu}=\sum_{\nu \rightarrow \mu} c_{\mu \nu} T_{\nu}
$$

and transform (4.9) to

$$
\begin{aligned}
& B_{\gamma} h_{m-1}\left[B_{\gamma}\right]=\sum_{\mu \vdash m} \frac{\Pi_{\mu}}{w_{\mu}} \frac{\tilde{H}_{\gamma}\left[M B_{\mu}\right]}{\Pi_{\gamma}} \sum_{\nu \rightarrow \mu} c_{\mu \nu} T_{\nu} \\
& =\sum_{\nu \vdash m-1} \frac{T_{\nu}}{w_{\nu}} \sum_{\mu \leftarrow \nu} \frac{\Pi_{\mu} \tilde{H}_{\gamma}\left[M B_{\mu}\right]}{\Pi_{\gamma}} c_{\mu \nu} \frac{w_{\nu}}{w_{\mu}} \\
& =\sum_{\nu \vdash m-1} \frac{T_{\nu}}{w_{\nu}} \sum_{\mu \leftarrow \nu} \frac{\Pi_{\mu} \tilde{H}_{\gamma}\left[M B_{\mu}\right]}{\Pi_{\gamma}} d_{\mu \nu} / M \\
& \text { (by reciprocity) }=\sum_{\nu \vdash m-1} \frac{T_{\nu}}{w_{\nu}} \sum_{\mu \leftarrow \nu} \tilde{H}_{\mu}\left[M B_{\gamma}\right] d_{\mu \nu} / M \\
& =\frac{h_{1}\left[M B_{\gamma}\right]}{M} \sum_{\nu \vdash m-1} \frac{T_{\nu}}{w_{\nu}} \tilde{H}_{\nu}\left[M B_{\gamma}\right]=B_{\gamma} h_{m-1}\left[\frac{M B_{\gamma}}{M}\right]
\end{aligned}
$$

which is plainly true, and completes the proof of (4.8).

An application of Proposition 4.1 yields another remarkable consequence of the action of the operator $D_{0}$. 
Theorem 4.1. Let $k \geq 0$. Recalling the notation in (1.16) for the Tesler matrix polynomials we have

$$
\left\langle\nabla e_{n+k}, h_{k} h_{1}^{n}\right\rangle=\sum_{\substack{a_{1}+a_{2}+\cdots+a_{n} \leq k \\ 0 \leq a_{i}}} P_{1+a_{1}, 1+a_{2}, \ldots, 1+a_{n}}(q, t) .
$$

Proof. An application of (4.1) with $m=n+k+1$ and $F=h_{k} h_{1}^{n}$ gives

$$
\left\langle\Delta_{e_{n+k}} e_{n+k}, h_{k} h_{1}^{n}\right\rangle=\left\langle\Delta_{e_{k} e_{1}^{n}} e_{n+k+1}, h_{n+k+1}\right\rangle .
$$

Now (2.26)f) gives

$$
\left\langle\Delta_{e_{k} e_{1}^{n}} e_{n+k+1}, h_{n+k+1}\right\rangle=\sum_{\mu \vdash n+k+1} \frac{M B_{\mu} \Pi_{\mu}}{w_{\mu}} e_{k}\left[B_{\mu}\right] B_{\mu}^{n}
$$

(by the second case of $(3.15))=\left(-\frac{1}{M}\right)^{n} \sum_{\mu \vdash n+k+1} \frac{M B_{\mu} \Pi_{\mu}}{w_{\mu}} e_{k}\left[B_{\mu}\right]\left(1-M B_{\mu}\right)^{n}$

$$
\begin{aligned}
= & \left(-\frac{1}{M}\right)^{n} \\
& \times\left.\sum_{\mu \vdash n+k+1} \frac{\tilde{H}_{\mu}[X]}{w_{\mu}} e_{k}\left[B_{\mu}\right]\left(1-M B_{\mu}\right)^{n}\right|_{X=M}
\end{aligned}
$$

$($ by $(2.13))=\left(-\frac{1}{M}\right)^{n} D_{0}^{n}$

$$
\begin{aligned}
& \times\left.\sum_{\mu \vdash n+k+1} \frac{\tilde{H}_{\mu}[X]}{w_{\mu}}\left\langle\tilde{H}_{\mu}, e_{k} h_{n+1}\right\rangle\right|_{X=M} \\
= & \left.\left(-\frac{1}{M}\right)^{n} D_{0}^{n} e_{n+1}\left[\frac{X}{M}\right] h_{k}\left[\frac{X}{M}\right]\right|_{X=M} .
\end{aligned}
$$

On the other hand an application of Proposition 3.1 for $a_{1}=a_{2}=\cdots=$ $a_{n}=0$ and $F=e_{n+1}\left[\frac{X}{M}\right] h_{k}\left[\frac{X}{M}\right]$ gives

$$
\begin{aligned}
& \left.D_{0}^{n} e_{n+1}^{*} h_{k}^{*}\right|_{X=M} \\
& =\left.e_{n+1}\left[1+\sum_{i=1}^{n} \frac{1}{z_{i}}\right] h_{k}\left[1+\sum_{i=1}^{n} \frac{1}{z_{i}}\right] \prod_{i=1}^{n} \Omega\left[-z_{i} M\right] \prod_{1 \leq i<j \leq n} \Omega\left[\frac{z_{i}}{z_{j}} M\right]\right|_{z_{1}^{0} z_{2}^{0} \cdots z_{n}^{0}} \\
& =\left.h_{k}\left[1+\sum_{i=1}^{n} \frac{1}{z_{i}}\right] \prod_{i=1}^{n} \Omega\left[-z_{i} M\right] \prod_{1 \leq i<j \leq n} \Omega\left[\frac{z_{i}}{z_{j}} M\right]\right|_{z_{1}^{1} z_{2}^{1} \cdots z_{n}^{1}} \\
& =\left.\sum_{\substack{a_{1}+a_{2}+\cdots+a_{n} \leq k \\
0 \leq a_{i}}} \prod_{i=1}^{n} \Omega\left[-z_{i} M\right] \prod_{1 \leq i<j \leq n} \Omega\left[\frac{z_{i}}{z_{j}} M\right]\right|_{z_{1}^{1+a_{1}} z_{2}^{1+a_{2}}+\cdots z_{n}^{1+a_{n}}}
\end{aligned}
$$




\section{From parking functions to Tesler matrices}

In a recent work P. Levande [12] establishes a beautiful weight preserving bijection between parking functions and Tesler matrices with hook sums $(1,1, \ldots, 1)$ and only one non zero element in each row. We will soon be deriving more general forms of Levande's results, and as a means to that end we include our own exposition of part of his work here, in notation compatible with previous sections of this paper. To state and prove his results we need to introduce some notation and establish a few auxiliary facts.

In our previous writings, parking functions were depicted as tableaux in the $n \times n$ lattice square as in the figure below.

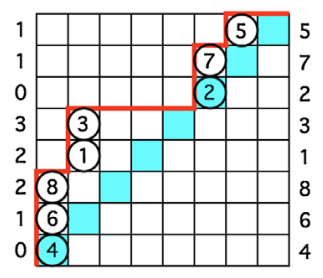

In this representation a parking function is constructed by first choosing a Dyck path, then placing circles in the cells adjacent to its NORTH steps and then filling the circles with $1,2, \ldots, n$ in a column increasing manner. The label $u_{i}$, on the left of row $i$, gives the number of cells between the NORTH step in that row and the diagonal. The label $v_{i}$ on the right of row $i$, gives the "car" in that row. In the computer a PF is stored as the two line array

$$
P F=\left[\begin{array}{llll}
v_{1} & v_{2} & \cdots & v_{n} \\
u_{1} & u_{2} & \cdots & u_{n}
\end{array}\right] .
$$

Thus, in the example above we obtain

$$
P F=\left[\begin{array}{llllllll}
4 & 6 & 8 & 1 & 3 & 2 & 7 & 5 \\
0 & 1 & 2 & 2 & 3 & 0 & 1 & 1
\end{array}\right]
$$

It is easy to see that a necessary and sufficient condition for such an array to represent a $\mathrm{PF}$ is that we have

$$
u_{1}=0, \quad u_{i} \leq u_{i-1}+1 \quad \text { with } u_{i}=u_{i-1}+1 \Longrightarrow v_{i}>v_{i-1} .
$$

Using this representation the two statistics "area" and "dinv" can be simply defined by setting 


$$
\begin{aligned}
& \operatorname{area}(P F)=\sum_{i=1}^{n} u_{i}, \\
& \operatorname{dinv}(P F)=\sum_{1 \leq i<j \leq n}\left(\chi\left(u_{i}=u_{j} \& v_{i}<v_{j}\right)+\chi\left(u_{i}=u_{j}+1 \& v_{i}>v_{j}\right)\right) .
\end{aligned}
$$

However historically this construction of a PF was only devised so as to assure that the corresponding "preference function" did "park the cars". In fact in the original definition a parking function for a one way street with $n$ parking spaces is a vector $f=\left(f_{1}, f_{2}, \ldots, f_{n}\right) \in[1, n]^{n}$ such that

$$
\#\left\{j: f_{j} \leq i\right\} \geq i \quad(\text { for all } 1 \leq i \leq n) .
$$

The simple bijection that converts the classical definition to its tableau form is simply to stack the cars as columns above the parking spaces they prefer to park. Thus in the example above car 1 wants to park in space 2 , car 2 wants to park in space 6 , etc. So the correponding preference function turns out to be

$$
p f=(2,6,2,1,7,1,6,1) .
$$

Of all the permutations we previously obtained from a parking function there are two more that we overlooked. Namely the one which gives the history or better, the time order in which the places are filled and the other gives the arrangement of the cars after they are finally all parked. It is easy to see that these permutations are inverses of each other, We will refer to the former as the "filling" permutation and the latter as the "parking" permutation.

For instance for the above parking function we have

$$
\text { filling }(p f)=(2,6,3,1,7,4,8,5), \quad \text { parking }(p f)=(4,1,3,6,8,2,5,7) \text {. }
$$

There is a simple algorithm for constructing filling $(p f)$ from the preference function $p f$. This is none other than an implementation of the way the parking takes place. We need only illustrate it in our particular example. Car 1 arrives and parks in 2, car 2 arrives and parks in 6 , but when car 3 arrives 2 is occupied and parks in 3. Car 4 arrives and parks in 1. Car 5 arrives and parks in 7 . But when car 6 arrives, who wants to park in 1 , finds 1, 2, 3 occupied, thus ends up parking in 4 since it is still free. The algorithm to get $\alpha=$ filling $(p f)$ from pf, starts by setting $\alpha_{1}=p f[1]$ then inductively having constructed the first $i-1$ components of $\alpha$ and given that $p f[i]=a$ 
we let $\alpha_{i}=a$ if $a$ is not in $\left(\alpha_{1}, \alpha_{2}, \ldots, \alpha_{i-1}\right)$ and let $\alpha_{i}=a+k+1$ if $a, a+1, \ldots, a+k$ are in $\left(\alpha_{1}, \alpha_{2}, \ldots, \alpha_{i-1}\right)$ and $a+k+1$ is not.

Finally, the permutation parking $(p f)$ is the inverse of filling $(p f)$ since filling $(p f)[i]$ gives where car $i$ parks or equivalently where we must place $i$ in $\operatorname{parking}(p f)$.

A classical beautiful fact in the theory of parking functions, which goes back to Kreweras is

Theorem 5.1. The inverse algorithm yields that for any $\alpha \in S_{n}$ the polynomial

$$
N_{\alpha}(q)=\sum_{f i l l i n g(p f)=\alpha} q^{\operatorname{area}(p f)}
$$

factors into a product of q-integers.

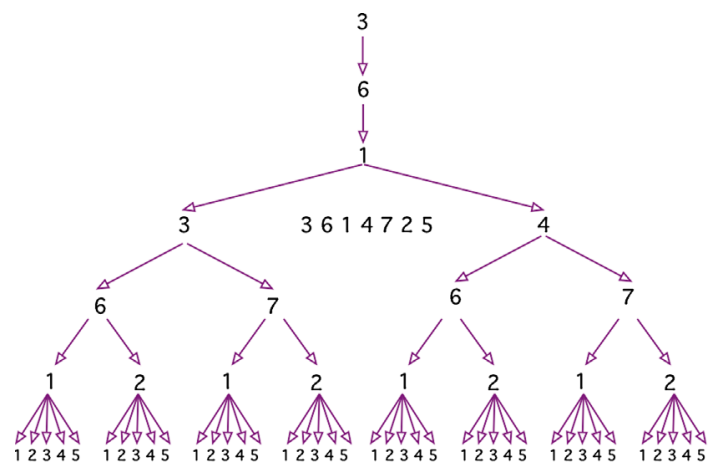

Proof. Let $\alpha=(3,6,1,4,7,2,5)$ be the given filling permutation. The tree depicted on the right gives all the preference functions that have $\alpha$ as their filling permutation. To see this, note that when car 1 arrives and parks at 3 it is clearly where it had wanted to park. The same is true for cars 2 and 3. But when car 4 arrives, and parks at 4 , maybe it wanted to park there, but since space 3 is occupied, it could very well have wanted to park at 3 . Similarly car 5 may have wanted to park at 7 or 6 , car 6 may have wanted to park at 2 or 1 and car 7 may have wanted to park at 5, 4,3,2 or 1 . More generally, if $\alpha_{s}=a$ and $a-1, a-2, \ldots, a-k$ are in the set $\left\{\alpha_{1}, \alpha_{2}, \ldots, \alpha_{s-1}\right\}$ but, $a-k-1$ is not, let us call the string $a, a-1, \ldots, a-k$ the "tail" of $\alpha_{s}$ otherwise, if that does not happen we will let $\alpha_{s}$ be its own tail. In any case we will refer to it as " $\operatorname{tail}\left(\alpha_{s}, \alpha\right)$ " or briefly "tail $\left(\alpha_{s}\right)$ ". This given, we see that the children of every node of the tree at level $s$ are to be labeled from right to left by the tail of $\alpha_{s}$. Moreover if we label the edges leading to the children of a node at level $s$, from right to left, by $1, q, q^{2}, \ldots, q^{k}$, then 
its is easy to see that the tree will have the further property that, as we go from the root down to a leaf, reading the labels of the successive nodes and multiplying the powers of $q$ encountered in the successive edges, we will obtain the preference function corresponding to that leaf together with $q$ to the power the extra travel that the cars end up traveling as a result of that preference. Note that the total amount of travel the cars end up doing after they enter the one way street is $\left(\begin{array}{c}n+1 \\ 2\end{array}\right)$ (in parking space units). Since the sum of the components of the preference function gives the amount of travel they were hoping to do, the difference, (which is none other than the area of the corresponding, Dyck path) is, in fact, the extra travel they were forced to do. This proves (5.1). Note that, for our example we also have the identity

$$
N_{(3,6,1,4,7,2,5)}(q)=[2]_{q}[2]_{q}[2]_{q}[5]_{q} .
$$

Likewise, in the general case, denoting by "\#S" the cardinality of a set $S$, we will have

$$
N_{\alpha}(q)=\prod_{s=1}^{n}\left[\# \operatorname{tail}\left(\alpha_{s}\right)\right]_{q}
$$

This proves our last assertion and completes the proof.

Levande proves that the same family of polynomials may be obtained from the subfamily of Tesler matrices in $\mathcal{T}[1,1, \ldots, 1]$ that have only one non-zero element in each row. Since in the $n \times n$ case this family has $n$ ! elements we will call these matrices "Permutational Tesler" or briefly "Permutational" and denote them " $\Pi[1,1, \ldots, 1]$ ".

Given a permutation $\alpha=\left(\alpha_{1}, \alpha_{2}, \ldots, \alpha_{n}\right)$, it will be convenient to call the smallest element $\alpha_{j}>\alpha_{s}$, that is to the right of $\alpha_{s}$, "the target of $\alpha_{s}$ in $\alpha$ ", and let $\alpha_{s}$ be its own target if all the elements to its right are smaller that it. In symbols

$$
\operatorname{target}\left[\alpha_{s}, \alpha\right]= \begin{cases}\alpha_{s} & \text { if } \max \left\{\alpha_{s+1}, \ldots, \alpha_{n}\right\}<\alpha_{s}, \\ \min \left\{\alpha_{j}: \alpha_{j}>\alpha_{s} \& j>s\right\} & \text { otherwise }\end{cases}
$$

This given Levande's result may be stated as follows.

Theorem 5.2. For each $n$ there is a bijection $\alpha \leftrightarrow A(\alpha)$ between $S_{n}$ and $\Pi[1,1, \ldots, 1]$ such that, if we set for each $A=\left\|a_{i, j}\right\|_{i, j=1}^{n}$

$$
W_{\Pi}[A]=\prod_{a_{i, j}>0}\left[a_{i, j}\right]_{q} .
$$


Then

$$
W_{\Pi}[A(\alpha)]=N_{\alpha}(q) \quad \forall \alpha \in S_{n}
$$

Proof. The construction of a matrix $A(\alpha) \in \Pi[1,1, \ldots, 1]$ satisfying (5.4) for each filling permutation $\alpha$ is immediate. In fact, we simply fill row $s$ by placing the integer \#tail $\left(\alpha_{s}\right)$ in column $j$, if the target of $\alpha_{s}$ is $\alpha_{j}$. Of course, if $\alpha_{s}$ is its own target then the integer \#tail $\left(\alpha_{s}\right)$ ends up in the diagonal.

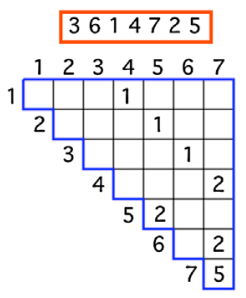

The reader will have no difficulty seeing that this recipe applied to the permutation $\alpha=(3,6,1,4,7,2,5)$ yields the permutational Tesler matrix on the right. Since this construction is easily shown to be reversible, and therefore injective, and the cardinalities are in both cases $n$ !, the only thing we need, to complete the proof, is to show that the resulting matrix is indeed in $\Pi[1,1, \ldots, 1]$ as asserted. This will be an immediate consequence of the following auxiliary fact.

Lemma 5.1. Suppose that for a given $\alpha=\left(\alpha_{1}, \alpha_{2}, \ldots, \alpha_{n}\right) \in S_{n}$ and some $1<k \leq n$ we have

$$
\begin{aligned}
& \left\{\alpha_{s}<\alpha_{k}: \text { target }\left[\alpha_{s}, \alpha\right]=\alpha_{k}\right\} \\
& \left.\quad=\left\{\alpha_{s_{1}}, \alpha_{s_{2}}, \ldots, \alpha_{s_{r}}\right\} \quad \text { (with } s_{1}<s_{2}<\cdots<s_{r}<k\right) .
\end{aligned}
$$

Then

$$
\operatorname{tail}\left(\alpha_{k}\right)=\left\{\alpha_{k}\right\}+\operatorname{tail}\left(\alpha_{s_{1}}\right)+\operatorname{tail}\left(\alpha_{s_{2}}\right)+\cdots+\operatorname{tail}\left(\alpha_{s_{r}}\right)
$$

the "+" denoting disjoint union.

Proof. Note that we must have $\alpha_{s_{1}}>\alpha_{s_{2}}>\cdots>\alpha_{s_{r}}$, since if for some $i$ we had $\alpha_{s_{i}}<\alpha_{s_{i+1}}$ then the target of $\alpha_{s_{i}}$ would be $\alpha_{s_{i+1}}$ and not $\alpha_{k}$. It follows from this that for each $i$ we must have mintail $\left(\alpha_{s_{i}}\right)>\alpha_{s_{i+1}}$. In fact, with the opposite inequality we would have $\min \operatorname{tail}\left(\alpha_{s_{i}}\right)<\alpha_{s_{i+1}}<\alpha_{s_{i}}$ and since $\operatorname{tail}\left(\alpha_{s_{i}}\right)$ is the interval of integers between its minimum and its maximum $\alpha_{s_{i}}$, this would force $\alpha_{s_{i+1}}$ to be in $\operatorname{tail}\left(\alpha_{s_{i}}\right)$ which contradicts 
$s_{i}<s_{i+1}$. Now suppose that for some $i$ we have $\alpha_{s_{i+1}}<\min \operatorname{tail}\left(\alpha_{s_{i}}\right)-1$, and set $a=\min \operatorname{tail}\left(\alpha_{s_{i}}\right)-1$. Clearly $a$ must be to the right of $\alpha_{s_{i}}$ in $\alpha$, for otherwise $a$ would in tail $\left(\alpha_{s_{i}}\right)$. But on the other hand $a$ can't be on the right of $\alpha_{s_{i+1}}$ for otherwise $\alpha_{s_{i+1}}$ would not have $\alpha_{k}$ as its target. Now $a$ cannot be its own target since it is less that $\alpha_{k}$ and to the left of it. Moreover $a$ cannot have $a_{k}$ as its target, and thus its target, say $b$, must be less than $\alpha_{k}$. However $b$ must be greater than $\alpha_{s_{i}}$ since all the elements in the interval $\left[a+1, \alpha_{s_{i}}\right]$ are in $\operatorname{tail}\left(\alpha_{s_{i}}\right)$. Since $b$ is is necessarily to the right of $\alpha_{s_{i}}$, this negates that $\alpha_{k}$ is the target of $\alpha_{s_{i}}$. This final contradiction proves that $\alpha_{s_{i+1}}=\min \operatorname{tail}\left(\alpha_{s_{i}}\right)-1$. This means that all the tails in (5.5) must be contiguous. To prove (5.5) it remains to show that

(1) 1 the entry min $\operatorname{tail}\left(\alpha_{s_{r}}\right)-1$ is not on the left of $\alpha_{k}$ and

(2) $2 \alpha_{s_{1}}=\alpha_{k}-1$.

Suppose that $a=\min \operatorname{tail}\left(\alpha_{s_{r}}\right)-1$ is to the left of $\alpha_{k}$, then, as in the previous argument $a$ must be to the right of $\alpha_{s_{r}}$ since otherwise it would be part of $\operatorname{tail}\left(\alpha_{s_{r}}\right)$. But then as before the target $b$ of $a$ is shown to be a number greater that $\alpha_{s_{r}}$, to the right of it and less than $\alpha_{k}$, and this negates that $\alpha_{k}$ is the target of $\alpha_{s_{i}}$. This contradiction proves (1).

Next let $a=\alpha_{k}-1$ and suppose that $\alpha_{s_{1}}<a$. But then $a$ can't be to the right of $\alpha_{s_{1}}$ for the $\alpha_{k}$ would not be the target of $\alpha_{s_{1}}$. But if it is to the left of $\alpha_{s_{1}}$ and therefore also to the left of $\alpha_{k}$, then $\alpha_{k}$ would be its target contradicting that $\alpha_{s_{1}}, \alpha_{s_{2}}, \ldots, \alpha_{s_{r}}$ are all the element of $\alpha$ that are less than $\alpha_{k}$ and have $\alpha_{k}$ as a target. This completes the proof of (5.5).

To complete the proof of Theorem 5.2, recall that the integer we place in row $k$ gives the size of the tail of $\alpha_{k}$. Thus the Tesler matrix condition for that row will be satisfied if and only if $\# \operatorname{tail}\left(\alpha_{k}\right)$ equals 1 plus the sum of the integers we placed in column $k$, Now if these integers were placed in rows $s_{1}<s_{2}<\cdots<s_{r}$ then this condition is simply

$$
\# \operatorname{tail}\left(\alpha_{k}\right)=1+a_{s_{1}, k}+a_{s_{2}, k}+\cdots+a_{s_{r}, k} .
$$

But by our construction, $\alpha_{s_{1}}, \alpha_{s_{2}}, \ldots, \alpha_{s_{r}}$ must have been the entries of $\alpha$ with target $\alpha_{k}$, thus for each $1 \leq i \leq r$ we must have set $a_{s_{i}, k}=\# \operatorname{tail}\left(\alpha_{s_{i}}\right)$. Thus (5.6) is none other than

$$
\# \operatorname{tail}\left(\alpha_{k}\right)=1+\# \operatorname{tail}\left(\alpha_{s_{1}}\right)+\# \operatorname{tail}\left(\alpha_{s_{2}}\right)+\cdots+\# \operatorname{tail}\left(\alpha_{s_{r}}\right),
$$

which is clearly an immediate consequence of (5.5). 


\section{Tesler matrices with arbitrary hook sums and weighted parking functions}

In $(1.16)$ we set

$$
P_{a_{1}, a_{2}, \ldots, a_{n}}(q, t)=\left(-\frac{1}{M}\right)^{n} \sum_{A \in \mathcal{T}\left(a_{1}, a_{2}, \ldots, a_{n}\right)} w_{H}(A)
$$

with

$$
w_{H}(A)=\prod_{i, j} h_{a_{i, j}}[-M] .
$$

Now it is easy to show that we have for any integer $k$

$$
h_{k}[-M]= \begin{cases}0 & \text { if } k<0 \\ 1 & \text { if } k=0 \\ -M[k]_{q t} & \text { if } k>0\end{cases}
$$

where

$$
[k]_{q t}=\frac{q^{k}-t^{k}}{q-t}=q^{k-1}+q^{k-2} q+\cdots+t^{k-2} q+t^{k-1} .
$$

Since as we have noted, when $a_{1}, a_{2}, \ldots, a_{n}$ are positive then all Tesler matrices in $\mathcal{T}\left(a_{1}, a_{2}, \ldots, a_{n}\right)$ have at least one non-zero element in each row, the identity in (6.2) assures that $P_{a_{1}, a_{2}, \ldots, a_{n}}(q, t)$ is a polynomial. In Section 4 we have seen a variety of cases where these polynomials are guaranteed to have positive integer coefficients. Computer experimentations have revealed a variety of other cases. In particular it is conjectured in [9] that the same thing happens when the $a_{1}, a_{2}, \ldots, a_{n}$ is any weakly increasing sequence.

In an effort to discover what collection of objects is $q, t$-enumerated by these polynomials, several values were obtained for $q=t=1$. The resulting data led the first author to conjecture (in a Banff meeting) that

$$
\begin{aligned}
P_{a_{1}, a_{2}, \ldots, a_{n}}(1,1)= & a_{1}\left(a_{1}+n a_{2}\right)\left(a_{1}+a_{2}+(n-1) a_{3}\right) \\
& \cdots\left(a_{1}+a_{2}+\cdots+a_{n-1}+2 a_{n}\right) .
\end{aligned}
$$

In this section we give two proofs of this conjecture. One uses an inductive argument and algebraic manipulations, while the other gives an explicit combinatorial interpretation of the conjecture by extending Levande's ideas. As 
a by-product we will obtain a parking function interpretation for the matrices $\Pi\left[a_{1}, a_{2}, \ldots, a_{n}\right]$. To see how this comes about we need some preliminary observations. Note first that setting $q=t=1$ in (6.1) reduces it to a sum over Permutational Tesler matrices. Now set

$$
Q_{a_{1}, a_{2}, \ldots, a_{n}}(q, t)=\sum_{A \in \Pi\left(a_{1}, a_{2}, \ldots, a_{n}\right)} w_{D}(A)
$$

with

$$
w_{D}(A)=\prod_{a_{i, j}>0}\left[a_{i, j}\right]_{q t} .
$$

Then, the identities in (6.2) imply that for all positive choices of $a_{1}, a_{2}, \ldots, a_{n}$ we have

$$
P_{a_{1}, a_{2}, \ldots, a_{n}}(1,1)=Q_{a_{1}, a_{2}, \ldots, a_{n}}(1,1) .
$$

Keeping this in mind, for a given parking function $P F \in \mathcal{P} \mathcal{F}_{n}$, let us set, for $p f=p f(P F)$

$$
y(p f)=\prod_{i=1}^{n} y_{\pi_{i}}
$$

where the $y_{i}$ 's are variables and the word $\pi=\pi_{1} \pi_{2} \cdots \pi_{n}$ is obtained by combining the preference function $p f$ corresponding to $P F$ with its parking permutation. More precisely, if $p f=(p f[1], p f[2], \ldots, p f[n])$ and $\operatorname{parking}(p f)=$ $\left(\beta_{1}, \beta_{2}, \ldots, \beta_{n}\right)$ then

$$
\pi_{i}=\beta_{p f[i]}
$$

Note further that it makes perfectly good sense to consider the matrices $\Pi\left[y_{1}, y_{2}, \ldots, y_{n}\right]$, In fact, for each of the $n$ ! choices of where to put the $n$ non zero entries in the rows of a permutational matrix, by proceeding from top to bottom we can simply place in row $s$ the sum of $y_{s}$ plus the entries in the previous rows that have been placed in column $s$. The entries of a matrix $A(y)=\left\|a_{i, j}\right\|_{i . j=1}^{n}$ are of course linear forms in $y_{1}, y_{2}, \ldots, y_{n}$ and we will set as before

$$
w(A(y))=\prod_{a_{i, j}(y) \neq 0} a_{i, j}(y) .
$$

This given we have the following fact 
Theorem 6.1. For each $n$ there is a bijection $\alpha \leftrightarrow A(\alpha, y)$ between $S_{n}$ and $\Pi\left[y_{1}, y_{2}, \ldots, y_{n}\right]$ such that

$$
\sum_{\text {filling }(p f)=\alpha} y(p f)=w(A(\alpha, y))
$$

Moreover we also have

$$
\begin{aligned}
\sum_{P F \in \mathcal{P F}_{n}} y(P F)= & \sum_{A(y) \in \Pi\left[y_{1}, y_{2}, \ldots, y_{n}\right]} w(A(\alpha, y)) \\
= & y_{1}\left(y_{1}+n y_{2}\right)\left(y_{1}+y_{2}+(n-1) y_{3}\right) \\
& \cdots\left(y_{1}+y_{2}+\cdots+y_{n-1}+2 y_{n}\right) .
\end{aligned}
$$

Proof. The identity in (6.11) is another consequence of Lemma 5.1. To begin, for a given $\alpha \in S_{n}$ and $\beta=\alpha^{-1}$ we construct the matrix $A(\alpha, y)$ by placing (for $s=1,2, \ldots, n$ ) in row $s$ and column $j$ the linear form

$$
a_{s, j}(y)=\sum_{a \in \operatorname{tail}\left(\alpha_{s}\right)} y_{\beta_{a}}
$$

if $\alpha_{j}$ is the target of $\alpha_{s}$ in $\alpha$. The same tree we used in the proof of Theorem 5.1 yields the identity

$$
\sum_{\text {filling }(p f)=\alpha} y(p f)=\prod_{s=1}^{n} \sum_{a \in \operatorname{tail}\left(\alpha_{s}\right)} y_{\pi_{a}} .
$$

Thus to complete the proof of (6.11) we need only verify that the resulting matrix $A(\alpha, y)$ lies in $\Pi\left[y_{1}, y_{2}, \ldots, y_{n}\right]$. For this we need that the linear form we place in row $k$ equals

$$
y\left[\beta_{\alpha_{k}}\right]+\sum_{a_{1} \in \operatorname{tail}\left(\alpha_{s_{1}}\right)} y\left[\beta_{a_{1}}\right]+\sum_{a_{2} \in \operatorname{tail}\left(\alpha_{s_{2}}\right)} y\left[\beta_{a_{2}}\right]+\cdots+\sum_{a_{r} \in \operatorname{tail}\left(\alpha_{s_{r}}\right)} y\left[\beta_{a_{r}}\right]
$$

where again $\alpha_{s_{1}}, \alpha_{s_{2}}, \ldots, \alpha_{s_{3}}$ are the entries of $\alpha$ whose target is $\alpha_{k}$. But this is again a consequence of applying $y[\beta]$ to every element of the identity in (5.5).

This given, the first equality in (6.12) follows from (6.11). To prove the second equality we can follow two different paths, either algebraic manipulations or direct combinatorial counting. We will do it both ways since each argument yields identities that may be useful in further work. We start with a simple combinatorialization of (6.4). 


\section{Proposition 6.1.}

$$
\sum_{P F \in \mathcal{P} \mathcal{F}_{n}} y(P F)=y_{1} \prod_{i=2}^{n}\left(y_{1}+y_{2}+\cdots+y_{i-1}+(n+2-i) y_{i}\right) .
$$

Proof. The monomial $y(P F)$ contributed by a parking function $P F$ to the sum on the left-hand side of (6.15) may be progressively obtained by the following steps as the cars arrive one by one. To begin, car 1 contributes the factor $y_{1}$. Having constructed the partial monomial $y_{j_{1}} y_{j_{2}} \cdots y_{j_{i-1}}$ after the arrival of cars $1,2, \ldots, i-1$, the factor $y_{j_{i}}$ contributed by car $i$ is $y_{i}$ if the parking space $p f[i]$ is free and is $y_{r}$ if space $p f[i]$ is already occupied by car $r$ there after car $i$ proceeds to the first free space.

To show that the polynomial resulting by summing these monomials factors as in (6.15), we make use of the original device of parking the cars on a circular one way street with $n+1$ parking spaces. Here again we construct each monomial as indicated above but from the unrestricted family of preference functions giving $n+1$ parking choices for each car. We have then $n+1$ choices for car 1 . For car 2, its choice could be the place where car 1 parked, in that case the partial monomial would be $y_{1}^{2}$. There remain $n$ free places where car 2 can park, in which case the partial monomial is $y_{1} y_{2}$. Now two spaces are occupied, one by car 1 and one by car 2 . So when car three arrives the possible new factors it can contribute are $y_{1}, y_{2}$ and in $n-1$ different ways $y_{3} \ldots$. We can clearly see now how the successive factors at the right-hand side of (6.15) do arise from this construction. Finally, when car $n$ comes cars $1,2, \ldots, n-1$ are already parked, and the final factor in the resulting monomial can be one of $y_{1}, y_{2}, \ldots, y_{n-1}$ and $y_{n}$ in two different ways. It follows that, by summing the monomials produced by all these $(n+1)^{n}$ preference functions, we must necessarily obtain the polynomial.

$$
(n+1) \times y_{1} \prod_{i=2}^{n}\left(y_{1}+y_{2}+\cdots+y_{i-1}+(n+2-i) y_{i}\right) .
$$

Because of the rotational symmetry of this particular parking algorithm, it follows that for each of the $n+1$ parking spaces, by summing the monomials coming from the preference functions that leave that space empty, we must invariably produce the same polynomial. Therefore by summing the monomials produced by the preference function that leave space $n+1$ empty we will obtain the polynomial in (6.16) divided by $n+1$. This proves (6.15) and completes the proof of (6.12) as well. 
We give next an inductive proof of (6.12).

\section{Proposition 6.2.}

$$
\sum_{A(y) \in \Pi\left[y_{1}, y_{2}, \ldots, y_{n}\right]} w(A(\alpha, y))=y_{1} \prod_{i=2}^{n}\left(y_{1}+y_{2}+\cdots+y_{i-1}+(n+2-i) y_{i}\right)
$$

Proof. Note first that the left-hand side of (6.7) for $n=2$ reduces to

$$
w\left[\begin{array}{cc}
y_{1} & 0 \\
0 & y_{2}
\end{array}\right]+w\left[\begin{array}{cc}
0 & y_{1} \\
0 & y_{1}+y_{2}
\end{array}\right]=y_{1} y_{2}+y_{1}^{2}+y_{1} y_{2}=y_{1}\left(y_{1}+2 y_{2}\right)
$$

Thus (6.17) holds true in this case. We can thus proceed by an induction argument, but for clarity and simplicity this idea is best understood in a special case. We will carry it out for $n=5$ and that will be sufficient. Let us denote the polynomial produced by the left-hand side of (6.17) by $L\left[y_{1}, y_{2}, \ldots, y_{n}\right]$.

Note that it immediately follows from the definition of the matrices in $\Pi\left[y_{1}, y_{2}, \ldots, y_{n}\right]$, by taking into account the column occupied by the nonzero element of the first row, that we must have the following recursion for $n=5$

$$
\begin{aligned}
L\left[y_{1}, y_{2}, y_{3}, y_{4}, y_{5}\right]= & y_{1} L\left[y_{2}, y_{3}, y_{4}, y_{5}\right]+y_{1} L\left[y_{1}+y_{2}, y_{3}, y_{4}, y_{5}\right] \\
& +y_{1} L\left[y_{2}, y_{1}+y_{3}, y_{4}, y_{5}\right]+y_{1} L\left[y_{2}, y_{3}, y_{1}+y_{4}, y_{5}\right] \\
& +y_{1} L\left[y_{2}, y_{3}, y_{4}, y_{1}+y_{5}\right] .
\end{aligned}
$$

Using MAPLE, the inductive hypothesis gives that these five L-polynomials are as listed in the display below

$$
\begin{gathered}
y_{2}\left(y_{2}+4 y_{3}\right)\left(y_{2}+y_{3}+3 y_{4}\right)\left(y_{2}+y_{3}+y_{4}+2 y_{5}\right) \\
\left(y_{1}+y_{2}\right)\left(y_{1}+y_{2}+4 y_{3}\right)\left(y_{1}+y_{2}+y_{3}+3 y_{4}\right)\left(y_{1}+y_{2}+y_{3}+y_{4}+2 y_{5}\right) \\
y_{2}\left(4 y_{1}+y_{2}+4 y_{3}\right)\left(y_{1}+y_{2}+y_{3}+3 y_{4}\right)\left(y_{1}+y_{2}+y_{3}+y_{4}+2 y_{5}\right) \\
y_{2}\left(y_{2}+4 y_{3}\right)\left(3 y_{1}+y_{2}+y_{3}+3 y_{4}\right)\left(y_{1}+y_{2}+y_{3}+y_{4}+2 y_{5}\right) \\
y_{2}\left(y_{2}+4 y_{3}\right)\left(y_{2}+y_{3}+3 y_{4}\right)\left(2 y_{1}+y_{2}+y_{3}+y_{4}+2 y_{5}\right) .
\end{gathered}
$$

Notice that the three middle polynomials have a common factor of $y_{1}+y_{2}+$ $y_{3}+y_{4}+2 y_{5}$, while the first and last start with the same three factors, while adding their last factors gives

$$
y_{2}+y_{3}+y_{4}+2 y_{5}+2 y_{1}+y_{2}+y_{3}+y_{4}+2 y_{5}=2\left(y_{1}+y_{2}+y_{3}+y_{4}+2 y_{5}\right) \text {. }
$$


Thus omitting the common factor $\left(y_{1}+y_{2}+y_{3}+y_{4}+2 y_{5}\right)$, combining the first and last polynomial in (6.18) and the remaining three polynomials we have

$$
\begin{gathered}
2 y_{2}\left(y_{2}+4 y_{3}\right)\left(y_{2}+y_{3}+3 y_{4}\right) \\
\left(y_{1}+y_{2}\right)\left(y_{1}+y_{2}+4 y_{3}\right)\left(y_{1}+y_{2}+y_{3}+3 y_{4}\right) \\
y_{2}\left(4 y_{1}+y_{2}+4 y_{3}\right)\left(y_{1}+y_{2}+y_{3}+3 y_{4}\right) \\
y_{2}\left(y_{2}+4 y_{3}\right)\left(3 y_{1}+y_{2}+y_{3}+3 y_{4}\right) .
\end{gathered}
$$

Now we see the same pattern emerging. Summing the last factors of the first and last in (6.19) gives

$$
2\left(y_{2}+y_{3}+3 y_{4}\right)+3 y_{1}+y_{2}+y_{3}+3 y_{4}=3\left(y_{1}+y_{2}+y_{3}+3 y_{4}\right)
$$

Omitting the common factor $\left(y_{1}+y_{2}+y_{3}+3 y_{4}\right)$ and combining the first and last now gives

$$
\begin{gathered}
3 y_{2}\left(y_{2}+4 y_{3}\right) \\
\left(y_{1}+y_{2}\right)\left(y_{1}+y_{2}+4 y_{3}\right) \\
y_{2}\left(4 y_{1}+y_{2}+4 y_{3}\right) .
\end{gathered}
$$

Now adding the last factors of the first and last gives

$$
\left.3\left(y_{2}+4 y_{3}\right)+4 y_{1}+y_{2}+4 y_{3}\right)=4\left(y_{1}+y_{2}+4 y_{3}\right)
$$

Omitting the factor $y_{1}+y_{2}+4 y_{3}$ and combining the first and last give

$$
\begin{gathered}
4 y_{2} \\
y_{1}+y_{2}
\end{gathered}
$$

whose sum is $y_{1}+5 y_{2}$ which is the last factor we needed to complete the induction and the proof.

\section{The weighted parking function setting for $q$-Teslers}

The problem that still remains open is to discover what combinatorial structures are $q, t$-counted by the polynomial in (1.16), namely

$$
P_{u_{1}, u_{2}, \ldots, u_{n}}(q, t)=\left(-\frac{1}{M}\right)^{n} \sum_{A \in \mathcal{T}\left(u_{1}, u_{2}, \ldots, u_{n}\right)} w_{H}(A)
$$


where we replaced the $a_{i}$ 's by the $u_{i}$ 's to avoid notational conflicts. Theorem 6.1, and more specifically the identity in (6.12), essentially gives a parking function setting for the polynomial $P_{a_{1}, a_{2}, \ldots, a_{n}}(1,1)$. In this section we go one step further towards the general open problem by working out the statistic that gives a parking function setting for the polynomial $P_{u_{1}, u_{2}, \ldots, u_{n}}(q, 1)$.

Note first that setting $t=1$ in (7.1) reduces it to the polynomial

$$
P_{u_{1}, u_{2}, \ldots, u_{n}}(q)=\sum_{A \in \Pi\left(u_{1}, u_{2}, \ldots, u_{n}\right)} w_{q}(A)
$$

with

$$
w_{q}(A)=\prod_{a_{i, j}>0}\left[a_{i, j}\right]_{q}
$$

We will next define the "q,u-weight" of a parking function $P F$ which we will denote " $w_{q, u}[P F]$ ". To this end, let $\alpha=\operatorname{filling}(P F)$ and $\beta=\operatorname{parking}(P F)$ and $p f$ be the corresponding preference function. Now recall that for a given $1 \leq i \leq n, \alpha_{i}$ gives the space that is occupied by car $i$. By definition $\operatorname{tail}\left[\alpha_{i}\right]$ gives the possible places that car $i$ may have prefered to park. Thus $p f[i]$ must necessarily be an element of $\operatorname{tail}\left[\alpha_{i}\right]$. More precisely, if tail $\left[\alpha_{i}\right]=$ $\left[\alpha_{i}-k_{i}, \ldots, \alpha_{i}-1, \alpha_{i}\right]$ then $p f[i]=\alpha_{i}-r_{i}$, for some $0 \leq r_{i} \leq k_{i}$. This given, let

$$
e x\left(\alpha, r_{i}\right)=\sum_{r_{i}+1 \leq j \leq k_{i}} u_{\beta_{\alpha_{i}-j}}
$$

and keeping in mind that, given that filling $(P F)=\alpha$, the preference function $p f$ as well as $P F$ are completely determined by the sequence $\left(r_{1}, r_{2}, \ldots, r_{n}\right)$, we set

$$
w_{q, u}[P F]=\prod_{i=1}^{n} q^{e x\left(\alpha, r_{i}\right)}\left[u_{\beta_{\alpha_{i}-r_{i}}}\right]_{q} .
$$

We are now in position to state and prove

Theorem 7.1. For every positive integral vector $u=\left(u_{1}, u_{2}, \ldots, u_{n}\right)$ we have

$$
\sum_{P F \in \mathcal{P} \mathcal{F}_{n}} w_{q, u}(P F)=P_{u_{1}, u_{2}, \ldots, u_{n}}(q) .
$$


Proof. Recall that a matrix $A=\left\|a_{i, j}\right\|_{i, j=1}^{n} \in \mathcal{U} \mathcal{P}$ is in $\Pi\left(u_{1}, u_{2}, \ldots, u_{n}\right)$ if and only if there is one non-zero element in its row and if the element in row $k$ is $a_{k, j}$ then given that the non-zero elements in column $k$ are

$$
a_{s_{1}, k}, a_{s_{1}, k}, \ldots, a_{s_{r}, k}
$$

we must have

$$
a_{k, j}=u_{k}+a_{s_{1}, k}+a_{s_{1}, k}+\cdots+a_{s_{r}, k}
$$

We will obtain all these matrices by a construction that follows closely the proof of Theorem 6.1. More precisely for a given $\alpha \in S_{n}$ we will let $A(\alpha)$ be a permutational matrix $A=\left\|a_{i, j}\right\|_{i, j=1}^{n} \in \mathcal{U P}$ obtained by placing, the non-zero element in row $s$, in column $k$ if $\alpha_{k}$ is the target of $\alpha_{s}$. But here, to satisfy the requirement in (7.4), we must set

$$
a_{s, k}=\sum_{a \in \operatorname{tail}\left(\alpha_{s}\right)} u_{\beta_{a}}
$$

with $\beta=\alpha^{-1}$. The reason for this is that if the non-zero elements in column $k$ are $a_{s_{1}, k}, a_{s_{2}, k}, \ldots, a_{s_{r}, k}$, then the identity in (5.5) gives

$$
\sum_{a \in \operatorname{tail}\left(\alpha_{k}\right)} u_{\beta_{a}}=u_{k}+a_{s_{1}, k}+a_{s_{2}, k}+\cdots+a_{s_{r}, k}
$$

which is (7.4) since the sum on the left-hand side is none other than that of the non-zero element we place in row $k$.

This identity gives that $A(\alpha) \in \Pi\left(u_{1}, u_{2}, \ldots, u_{n}\right)$ for all $\in S_{n}$ and since this construction is reversible thus injective and the cardinalities are the same, it must also be surjective.

Note next that it follows from our definition in (7.4) that for a fixed $1 \leq i \leq n$ we have, for $\operatorname{tail}\left[\alpha_{i}\right]=\left[\alpha_{i}-k_{i}, \ldots, \alpha_{i}-1, \alpha_{i}\right]$

$$
\begin{aligned}
& \sum_{r_{i}=0}^{k_{i}} q^{e x\left(\alpha, r_{i}\right)}\left[u_{\beta_{\alpha_{i}-r_{i}}}\right]_{q} \\
& =\left[u_{\beta_{\alpha_{i}-k_{i}}}\right]_{q}+q^{u_{\beta_{\alpha_{i}-k_{i}}}}\left[u_{\beta_{\alpha_{i}-k_{i}+1}}\right]_{q} \\
& +q^{u_{\beta_{\alpha_{i}-k_{i}}}+u_{\beta_{\alpha_{i}-k_{i}+1}}}\left[u_{\beta_{\alpha_{i}-k_{i}+2}}\right]_{q}+\cdots+q^{u_{\beta_{\alpha_{i}-k_{i}}}+\cdots+u_{\beta_{\alpha_{i}-1}}}\left[u_{\beta_{\alpha_{i}}}\right]_{q} \\
& =\left[\sum_{a \in \operatorname{tail}\left[\alpha_{i}\right]} u_{\beta_{a}}\right]_{q}=\left[a_{i, j_{i}}(\alpha)\right]_{q} \text {, }
\end{aligned}
$$


where the last equality follows from the definition in (7.9) provided that the non-zero element in row $i$ of the matrix $A(\alpha)$ is in column $j_{i}$. Thus it follows from (7.5) that

$$
\sum_{\text {filling }(P F)=\alpha} w_{q, u}[P F]=\prod_{i=1}^{n}\left(\sum_{r_{i}=0}^{k_{i}} q^{e x\left(\alpha, r_{i}\right)}\left[u_{\beta_{\alpha_{i}-r_{i}}}\right] q\right)=w_{q}[A(\alpha)]
$$

and the equality in (7.6) is then obtained by summing over all $\alpha \in S_{n}$. This completes our proof.

The special case $t=1 / q$ of the bigraded Hilbert series for diagonal harmonics is known to have a nice formulation, which in the language of (6.1) can be expressed as

$$
q^{\left(\begin{array}{l}
n \\
2
\end{array}\right)} P_{(1,1, \ldots, 1)}(q, 1 / q)=\left(1+q+q^{2}+\cdots+q^{n}\right)^{n-1} .
$$

Maple calculations indicate that this nice special case extends in the following elegant way.

Conjecture 7.1. Let $\left(a_{1}, a_{2}, \ldots, a_{n}\right)$ be an arbitrary sequence of positive integers, and set

$$
F=\sum_{i=1}^{n}(n+1-i) a_{i}-n
$$

Then

$$
q^{F} P_{\left(a_{1}, a_{2}, \ldots, a_{n}\right)}(q, 1 / q)=\left[a_{1}\right]_{q^{n+1}} \prod_{i=2}^{n}\left[\sum_{j=1}^{i-1} a_{j}+(n+2-i) a_{i}\right]_{q} .
$$

\section{The Dyck path associated to a Tesler matrix}

In this section we show how to associate a Dyck path to a Tesler matrix in $\mathcal{T}[1,1,1, \ldots, 1]$, which leads to "prime parking functions" and refinements of some of our results and conjectures on Tesler matrices.

Let us say that an $n \times n$ matrix $A=\left\|a_{i, j}\right\|_{i, j=1}^{n}$ factors at $1 \leq k \leq n-1$ if it is block diagonal with an initial block of size $k \times k$. More precisely, $A$ factors at $k$ if and only if $a_{i, j}=0$ when $1 \leq i \leq k \& j>k$ and when $i>k \& 1 \leq j \leq k$; in that case, the two submatrices $A_{1}=\left\|a_{i, j}\right\|_{i, j=1}^{k}$ and $A_{2}=\left\|a_{i, j}\right\|_{k<i, j \leq n}$ will be referred to as "factors" of $A$. It develops that a very simple factorization criterion follows in full generality straight from the definition of Tesler matrices. 
Proposition 8.1. For any Tesler matrix $A=\left\|a_{i, j}\right\|_{i, j=1}^{n} \in \mathcal{T}\left[u_{1}, u_{2}, \ldots, u_{n}\right]$ we have the equality

$$
\sum_{s=1}^{k} a_{s, s}+\sum_{i=1}^{k} \sum_{j=k+1}^{n} a_{i, j} \chi(i<j)=\sum_{s=1}^{k} u_{s} \quad(\text { for all } 1 \leq k \leq n) .
$$

Thus we have the inequality

$$
\sum_{s=1}^{k} a_{s, s} \leq \sum_{s=1}^{k} u_{s}
$$

with equality for $k=n$. Moreover, we see that $A$ factors at $k$ if and only if

$$
\sum_{s=1}^{k} a_{s, s}=\sum_{s=1}^{k} u_{s}
$$

Proof. Note that the hook sum equalities for a Tesler matrix may be written in the form

$$
\sum_{j=1}^{n} a_{s, j} \chi(j \geq s)-\sum_{i=1}^{n} a_{i, s} \chi(i<s)=u_{s} .
$$

Summing for $1 \leq s \leq k$ gives

$$
\sum_{s=1}^{k} a_{s, s}+\sum_{j=1}^{n} \sum_{s=1}^{k} a_{s, j} \chi(j>s)-\sum_{i=1}^{n} \sum_{s=1}^{k} a_{i, s} \chi(i<s)=\sum_{s=1}^{k} u_{s}
$$

Replacing $s$ by $i$ in the first sum and $s$ by $j$ in the second sum gives

$$
\sum_{s=1}^{k} a_{s, s}+\sum_{i=1}^{k} \sum_{j=1}^{n} a_{i, j} \chi(i<j)-\sum_{i=1}^{k} \sum_{j=1}^{k} a_{i, j} \chi(i<j)=\sum_{s=1}^{k} u_{s}
$$

and (8.1) follows by canceling equal terms. But now we can clearly see that (8.2) can hold if and only if

$$
\sum_{i=1}^{k} \sum_{j=k+1}^{n} a_{i, j} \chi(i<j)=0 .
$$

Thus the last assertion is a immediate consequence of the non-negativity of the entries of Tesler matrices. 
The inequality in (8.2) allows us to associate to each matrix in $\mathcal{T}[1,1, \ldots, 1]$ a Dyck path by the following construction. Given $A=$ $\left\|a_{i, j}\right\|_{i, j=1}^{n} \in T[1,1, \ldots, 1]$, starting at $(0,0)$ of the $n \times n$ lattice square, and for each diagonal element $a_{i, i}$, we go successively NORTH one unit step and then EAST $a_{i, i}$ unit steps. The inequality in (8.2) (when all $u_{i}=1$ ) assures that the resulting path remains weakly above the diagonal $(i, i)$. Moreover, since each time equality holds in (8.2), the factorization criterion applies, and there is a direct connection between the Dyck path and the block diagonal structure of the corresponding matrix.

In particular it follows from this that the Tesler matrix does not factor at any $k$ if and only if its Dyck path never hits the diagonal. Computer calculations indicate that the weighted sum of these matrices yields a polynomial with positive coefficients, whose evaluation at $t=q=1$ appears to be enumerating structures equi-numerous with the prime parking functions of Novelli-Thibon [14]. Further explorations with the Tesler matrices in $\mathcal{T}[1,1, \ldots, 1]$ led to a variety of other conjectures relating the shape of the Dyck paths to the combinatorial properties of the sum of their weights. To state these findings we need some auxiliary facts and definitions.

To begin, given a preference function $f=\left(f_{1}, f_{2}, \ldots, f_{n}\right)$, we will say that it factors at $1 \leq k<n$ if $\max \left\{f_{i}: 1 \leq i \leq k\right\}=k$ and $\min \left\{f_{i}\right.$ : $k+1 \leq i \leq n\}=k+1$. If that happens we say $f$ itself may be expressed as a "product" by setting $f=g \oplus h$ with $g=\left(f_{1}, f_{2}, \ldots, f_{k}\right)$ and $h=$ $\left(f_{k+1}-k, f_{k+2}-k, \ldots, f_{n}-k\right)$. Since both $g$ and $h$ are necessarily preference functions for one way streets with $k$ and $n-k$ spaces respectively, we are led in this manner to a unique factorization of each preference function into a "product" of "prime" preference functions. Denote by $\pi_{n}$ the number of prime preference functions $f=\left(f_{1}, f_{2}, \ldots, f_{n}\right)$. Using the unique factorization, together with the fact that $(n+1)^{n-1}$ gives the total number of preference functions $f=\left(f_{1}, f_{2}, \ldots, f_{n}\right)$, Novelli and Thibon derive that

$$
\begin{aligned}
& \sum_{n \geq 1} \pi_{n} t^{n}=1-\frac{1}{1+\sum_{n \geq 1}(n+1)^{n-1} t^{n}} \\
& (8.4) \quad=t+2 t^{2}+11 t^{3}+92 t^{4}+1014 t^{5}+13795 t^{6}+223061 t^{7}+O\left(t^{8}\right) .
\end{aligned}
$$

Recall that we have defined the "diagonal composition" of a Dyck path $D$ and have denoted it " $p(D)$ " the length of the intervals between successive diagonal hits. Note that for a Dyck path in the $n \times n$ lattice square that returns to the diagonal only at the end we will set $p(D)=(n)$. 
Conjecture 8.1. For each composition $p \mid=n$ the sum

$$
Q_{p}(q, t)=\sum_{A \in \mathcal{T}[1,1, \ldots, 1]} w_{H}(A) \chi(p(\operatorname{Dyck}(A))=p)
$$

evaluates to a polynomial with positive integer coefficients.

We now show that when $q=t=1$, "prime" Tesler matrices have a nice generating function, which can be understood by the properties of "filling" permuations. Let us say that an element $\alpha_{s}$ of a permutation $\alpha=\left(\alpha_{1}, \alpha_{2}, \ldots, \alpha_{n}\right)$ is "salient" if all the elements to its right are smaller. Let us say that $\alpha$ "factors" at $k$, if the elements $\alpha_{1}, \alpha_{2}, \ldots, \alpha_{k}$ are a permutation of $n, n-1, \ldots, n-k+1$. Note that in this case we may express $\alpha$ as a "juxtaposition product" $\beta \otimes \gamma$, of a permutation $\beta \in S_{k}$ by a permutation $\gamma \in S_{n-k}$, by simply letting $\beta$ be the permutation obtained by subtracting $n-k$ from $\alpha_{1}, \alpha_{2}, \cdots, \alpha_{k}$ and taking $\gamma=\left(\alpha_{k+1} \alpha_{k+2}, \ldots, \alpha_{n}\right)$. Let us say that a permutation $\alpha \in S_{n}$ is "prime" if and only if it does not factor at any $1 \leq k \leq n-1$. Finally define the "weight" of a permutation $\alpha \in S_{n}$, denoted " $w(\alpha)$ ", to be the product of the sizes of all the tails of its elements. In symbols

$$
w(\alpha)=\prod_{s=1}^{n} \# \operatorname{tail}\left(\alpha_{s}\right)
$$

Note that this weight is multiplicative, that is we have

$$
w(\beta \otimes \gamma)=w(\beta) \times w(\gamma)
$$

This given, let us set

$$
G(t)=\sum_{n \geq 1} t^{n} \sum_{\alpha \in S_{n}} w(\alpha) \chi(\alpha \text { prime }) .
$$

Theorem 5.2 has the following immediate corollary.

\section{Proposition 8.2.}

$$
G(t)=1-\frac{1}{1+\sum_{n \geq 1}(n+1)^{n-1} t^{n}} .
$$

Proof. Setting $q=1$ in (5.2) and summing over $\alpha \in S_{n}$ Theorem 5.1 gives

$$
\sum_{\alpha \in S_{n}} w(\alpha)=\# \mathcal{P} \mathcal{F}_{n}=(n+1)^{n-1}
$$


Thus the weighted generating function of permutations is the formal power series

$$
F(t)=1+\sum_{n \geq 1}(n+1)^{n-1} t^{n}
$$

since each permutation can be uniquely factorized as a product of prime permutations, the multiplicativity of the weight function gives

$$
F(t)=1+G(t)+G(t)^{2}+G(t)^{3}+\cdots=\frac{1}{1-G(t)}
$$

and (8.10) follows by solving for $G(t)$.

\section{Corollary 8.1.}

$$
\sum_{n \geq 1} N_{(n)}(1,1) t^{n}=1-\frac{1}{1+\sum_{n \geq 1}(n+1)^{n-1} t^{n}} .
$$

The connection between parking functions and permutational Tesler matrices $\Pi[1,1, \ldots, 1]$ offered by Theorem 5.2 leads to the following result.

Proposition 8.3. The matrix $A(\alpha, y)=\left\|a_{i, j}(\alpha)\right\|_{i, j=1}^{n} \in \Pi\left[y_{1}, y_{2}, \ldots, y_{n}\right]$ corresponding to a permutation $\alpha \in S_{n}$ factors at $k$ if and only if $\alpha$ itself factors at $k$ and that happens if and only if

$$
a_{1,1}(\alpha)+\alpha_{2}(\alpha)+\cdots+a_{k, k}(\alpha)=y_{1}+y_{2}+\cdots+y_{k} .
$$

Proof. We have seen that $A(\alpha)$ factors at $k$ if and only if (8.13) holds true. Thus we need only show the first statement.

Recall, setting $\beta=\alpha^{-1}$ (see (6.13)), that we construct the matrix $A(\alpha, y)$ by placing in row $s$ and column $j$ the linear form

$$
a_{s, j}(\alpha, y)=\sum_{a \in \operatorname{tail}\left(\alpha_{s}\right)} y_{\beta_{a}}
$$

provided $\alpha_{j}$ is the target of $\alpha_{s}$. Thus $A(\alpha, y)$ factors at $k$ if and only if, for all $1 \leq s \leq k$ the target of $\alpha_{s}$ occurs to the left of $\alpha_{k+1}$. In particular $\alpha_{k}$ must be its own target and therefore in this case the non zero element in row $k$ lies in the diagonal. More generally in any matrix $A(\alpha, y)$ the diagonal non zero elements correspond to the self-targeting $\alpha_{s}$. In other words we have $a_{s, s} \neq 0$ if and only if $\alpha_{s}$ is salient in $\alpha$. Now suppose that $A(\alpha, y)$ 
factors at $k$ and that $\alpha_{s_{1}}>a_{s_{2}}>\cdots>\alpha_{s_{r}}=\alpha_{k}$ are the salient elements of $\alpha$ in $\left\{\alpha_{1}, \alpha_{2}, \ldots, a_{k}\right\}$. Of course we must have $s_{1}<s_{2}<\cdots<\alpha_{s_{r}}$. Let $\operatorname{tail}\left(\alpha_{s_{i}}\right)=\left\{a_{s_{i}}, a_{s_{i}}-1, \ldots, a_{s_{i}}-k_{i}\right\}$. Since all the element of $\operatorname{tail}\left(\alpha_{s_{i}}\right)$ are to the left of $\alpha_{s_{i}}$ and a fortiori to the left of $\alpha_{s_{i+1}}$ we cannot have $\alpha_{s_{i+1}} \in \operatorname{tail}\left(\alpha_{s_{i}}\right)$. This gives $\alpha_{s_{i+1}} \leq a_{s_{i}}-k_{i}-1$. We claim that we must have equality here. Suppose if possible that $\alpha_{s_{i+1}}<a_{s_{i}}-k_{i}-1$. Then $a_{s_{i}}-k_{i}-1$ cannot be to the left of $\alpha_{s_{i}}$ for otherwise it would be in its tail. Nor it can be to the right of $\alpha_{s_{i+1}}$ since it is greater than $\alpha_{s_{i+1}}$. Since $a_{s_{i}}-k_{i}-1$ is not not one of the salients of $\alpha$ in $\alpha_{1}, \alpha_{2}, \ldots, a_{k}$ its target must be to its right. This target must be greater than $\alpha_{s_{i}}$ since all the entries in the interval $\left[\alpha_{s_{i}}-k_{i}, \alpha_{s_{i}}\right]$ are to the left of $a_{s_{i}}-k_{i}-1$ but that contradicts the saliency of $\alpha_{s_{i}}$. This proves that $\alpha_{s_{i+1}}=a_{s_{i}}-k_{i}-1$. Consequently the tails are disjoint contiguous intervals of integers. Thus

$$
\operatorname{tail}\left(\alpha_{s_{1}}\right)+\operatorname{tail}\left(\alpha_{s_{2}}\right)+\cdots+\operatorname{tail}\left(\alpha_{s_{r}}\right)=\left[\alpha_{s_{r}}-k_{r}, \alpha_{s_{1}}\right]
$$

and since the first salient element of a permutation of $S_{n}$ is necessarily $n$ it follows that

$$
\left[\alpha_{s_{r}}-k_{r}, \alpha_{s_{1}}\right]=\left[\alpha_{s_{r}}-k_{r}, n\right]
$$

Since all the elements to the right of $\alpha_{s_{r}}$ must be less that $\alpha_{s_{r}}$ and therefore also less than $\alpha_{s_{r}}-k_{r}$ it follows that $\alpha_{1}, \alpha_{2}, \ldots, \alpha_{k}$ is a permutation of the interval $\left[\alpha_{s_{r}}-k_{r}, n\right]$. This shows that $\alpha$ itself factors at $k$. Moreover, (8.14) gives

$$
a_{s_{1}, s_{1}}+a_{s_{2}, s_{2}}+\cdots+a_{s_{r}, s_{r}}=\sum_{i=1}^{k} y_{\beta_{\alpha_{i}}}=\sum_{i=1}^{k} y_{i} .
$$

Proving (8.13). Conversely, if (8.13) holds true, then assuming again that $\alpha_{s_{1}}>a_{s_{2}}>\cdots>\alpha_{s_{r}}=\alpha_{k}$ are the salient elements of $\alpha$ in $\left\{\alpha_{1}, \alpha_{2}, \ldots, a_{k}\right\}$, we must have

$$
a_{s_{1}, s_{1}}+a_{s_{2}, s_{2}}+\cdots+a_{s_{r}, s_{r}}=\sum_{i=1}^{k} y_{i}
$$

and (8.16) gives that

$$
\operatorname{tail}\left(\alpha_{s_{1}}\right)+\operatorname{tail}\left(\alpha_{s_{2}}\right)+\cdots+\operatorname{tail}\left(\alpha_{s_{r}}\right)=\left\{\alpha_{1}, \alpha_{2}, \ldots, \alpha_{k}\right\} .
$$

A reverse of the previous argument gives that $\alpha$ factors at $k$. The reader should have no difficulty filling in the omitted details. 
As an immediate corollary of Proposition 8.3 we have

Theorem 8.1. Call the matrices $A \in \Pi[1,1, \ldots, 1]$ ( $n$ ones) that do not factor "prime". Then if we set $w_{\Pi}[A]=\prod_{a_{i, j}>0} a_{i, j}$ we have

$$
\sum_{A \in \Pi[1,1, \ldots, 1]} w_{\Pi}[A] \chi(\text { A prime })=\sum_{\alpha \in S_{n}} w(\alpha) \chi(\alpha \text { prime }) .
$$

In particular, Propositions 8.2 and 8.4 show that the resulting integer counts the number of parking functions in $\mathcal{P F}_{n}$ that are prime in the sense of Novelli-Thibon.

\section{References}

[1] F. Bergeron and A. M. Garsia, Science Fiction and Macdonald's Polynomials, Algebraic Methods and q-Special Functions (Montréal, QC, 1996), CRM Proc. Lecture Notes, vol. 22, Amer. Math. Soc., Providence, RI, 1999, pp. 1-52. MR1726826

[2] F. Bergeron, A. M. Garsia, M. Haiman, and G. Tesler, Identities and positivity conjectures for some remarkable operators in the theory of symmetric functions, Methods in Appl. Anal. 6 (1999), 363-420. MR1803316

[3] A. M. Garsia and J. Haglund, A proof of the q, t-Catalan positivity conjecture, Discrete Math. 256 (2002), 677-717. MR1935784

[4] A. M. Garsia and M. Haiman, A remarkable q, t-Catalan sequence and q-Lagrange inversion, J. Algebraic Combin. 5, no. 3 (1996), 191-244. MR1394305

[5] A. Garsia, M. Haiman and G. Tesler, Explicit Plethystic Formulas for the Macdonald q, t-Kostka Coefficients, Séminaire Lotharingien de Combinatoire, B42m (1999), 45 pp. MR1701592

[6] A. M. Garsia and G. Tesler, Plethystic formulas for Macdonald q, tKostka coefficients, Adv. Math. 123 (1996), 144-222. MR1420484

[7] J. Haglund, A proof of the q, t-Schröder conjecture, Internat. Math. Res. Notices 11 (2004), 525-560. MR2038776

[8] J. Haglund, The q, t-Catalan Numbers and the Space of Diagonal Harmonics, AMS University Lecture Series, vol. 41 (2008), p. 167. $\operatorname{MR} 2371044$ 
[9] J. Haglund, A polynomial expression for the Hilbert series of the quotient ring of diagonal coinvariants, Adv. Math. 227 (2011), 2092-2106. MR2803796

[10] J. Haglund, M. Haiman, N. Loehr, J. B. Remmel, and A. Ulyanov, A combinatorial formula for the character of the diagonal coinvariants, Duke J. Math. 126 (2005), 195-232. MR2115257

[11] J. Haglund and N. Loehr, A conjectured combinatorial formula for the Hilbert series for diagonal harmonics, in: Proceedings of the FPSAC Conference, Melbourne, Australia, 2002, Discrete Math. 298 (2005) 189-204. MR2163448

[12] M. Haiman, Vanishing theorems and character formulas for the Hilbert scheme of points in the plane, Invent. Math. 149 (2002), 371-407. MR1918676

[13] P. Levande, Special cases of the parking functions conjecture and upper-triangular matrices, Discrete Math. Theor. Comput. Sci., in: Proceedings of the 23rd Internat. Conf. on Formal Power Series and Algebraic Combinatorics (FPSAC 2011), pp. 635-644. See www.dmtcs.org/dmtcs-ojs/index.php/proceedings/issue/view/119. MR2820747

[14] I. G. Macdonald, Symmetric Functions and Hall Polynomials, Oxford Mathematical Monographs, 2nd ed., Oxford Science Publications, The Clarendon Press, Oxford University Press, New York, 1995. MR1354144

[15] J.-C. Novelli and J.-Y. Thibon, Hopf algebras and Dendriform structures arising from parking functions, Fund. Math. 193 (2007) 189-241. MR2289770

D. Armstrong

Department of Mathematics

UNIVERSITY OF MiAmi

Coral Gables, FL 33124-4250

USA

E-mail address: d.armstrong@math.miami.edu

\author{
A. Garsia \\ Department of Mathematics \\ University of California at San Diego \\ LA Jolla, CA 92093-0112 \\ USA \\ E-mail address: garsia@math.ucsd.edu
}


J. HAGLUND

Department of Mathematics

University of Pennsylvania

PhiladelPhia, PA 19104-6395

USA

E-mail address: jhaglund@math.upenn.edu

B. RHOADES

Department of Mathematics

University OF SOUTHERN CALIFORNiA

Los Angeles, CA 90089

USA

E-mail address: brhoades@usc.edu

B. SAGAN

Department of Mathematics

Michigan State University

EAST LANSING, MI 48824-1027

USA

E-mail address: sagan@math.msu.edu

Received November 16, 2011 